\title{
Random Access Game and Medium Access Control Design
}

\author{
Lijun Chen, Member, IEEE, Steven H. Low, Fellow, IEEE, and John C. Doyle
}

\begin{abstract}
Motivated partially by a control-theoretic viewpoint, we propose a game-theoretic model, called random access game, for contention control. We characterize Nash equilibria of random access games, study their dynamics, and propose distributed algorithms (strategy evolutions) to achieve Nash equilibria. This provides a general analytical framework that is capable of modeling a large class of system-wide quality-of-service $(\mathrm{QoS})$ models via the specification of per-node utility functions, in which system-wide fairness or service differentiation can be achieved in a distributed manner as long as each node executes a contention resolution algorithm that is designed to achieve the Nash equilibrium. We thus propose a novel medium access method derived from carrier sense multiple access/collision avoidance (CSMA/CA) according to distributed strategy update mechanism achieving the Nash equilibrium of random access game. We present a concrete medium access method that adapts to a continuous contention measure called conditional collision probability, stabilizes the network into a steady state that achieves optimal throughput with targeted fairness (or service differentiation), and can decouple contention control from handling failed transmissions. In addition to guiding medium access control design, the random access game model also provides an analytical framework to understand equilibrium and dynamic properties of different medium access protocols.
\end{abstract}

Index Terms-Contention-based medium access, control-theoretic analysis, game theory, Nash equilibrium, strategy update mechanism.

\section{INTRODUCTION}

W IRELESS channel is a shared medium that is interference-limited. Contention-based medium access control (contention control) is a distributed strategy to access and share wireless channel among contending wireless nodes. From a control-theoretic point of view, it consists of two components: a contention resolution algorithm that dynamically adjusts channel access probability in response to contention in the network and a feedback mechanism that updates a contention measure and sends it back to wireless nodes. Contention resolution is usually achieved through two mechanisms: persistence and backoff [1]. In the persistence mechanism, each wireless node maintains a persistence probability and accesses the channel with this probability when it perceives an idle channel. In the backoff mechanism, channel access probability is implemented through a backoff algorithm, and each wireless

Manuscript received August 20, 2008; revised May 21, 2009; approved by IEEE/ACM TRANSACTIONS ON NETWORKING Editor R. Mazumdar. First published February 17, 2010; current version published August 18, 2010.

The authors are with the Engineering and Applied Science Division, California Institute of Technology, Pasadena, CA 91125 USA (e-mail: chen@ cds. caltech.edu; slow@caltech.edu; doyle@cds.caltech.edu).

Color versions of one or more of the figures in this paper are available online at http://ieeexplore.ieee.org.

Digital Object Identifier 10.1109/TNET.2010.2041066 node maintains a contention window and waits for a random amount of time bounded by the contention window before a transmission. When simultaneous accesses to the channel by different nodes cause contention, the persistence probability or contention window is adjusted appropriately so that contention is reduced. Different medium access control methods differ in terms of how they adjust these parameters in response to contention and what contention measure they use. For example, the standard IEEE 802.11 DCF uses a backoff mechanism and a binary contention signal-packet collision or successful transmission - in which each wireless node doubles its contention window upon a collision (binary exponential backoff) and sets it to the base value upon a successful transmission [2].

The choice of contention measure and contention resolution algorithm is key to the performance of medium access methods. "Inappropriate" choice of these two components will result in poor performance. For example, in high-load scenarios, 802.11 DCF results in excessive collisions and, hence, low throughput because setting to the base contention window upon successful transmission is too drastic and each new transmission starts with the base contention window independent of the contention level in the network. It also has short-term unfairness problem due to oscillation in the contention window. The binary exponential backoff directly causes short-term unfairness. However, this oscillation in the contention window is unavoidable because DCF uses a binary contention signal. In order to achieve high efficiency (high throughput and low collision) and better fairness, we need to stabilize the network into a steady state that sustains an appropriate contention window size (or equivalently, channel access probability) for each node. Furthermore, how we can estimate and implement the contention measure is important. Almost all medium access methods, including 802.11 DCF, adapt to packet collisions. However, they cannot distinguish collisions from corrupted frames that are common in wireless networks. This leads to increased unfairness and lower throughput. To ensure good performance, we need to use a contention measure whose estimation is not based on packet collisions and decouple contention control from handling failed transmissions.

The main motivation of this work is to provide an analytical framework to systematically study contention control and design medium access methods that could stabilize the network around a steady state with a target fairness (or service differentiation) and high efficiency. To this end, we define a general game-theoretic model, called random access game, to capture the interaction among wireless nodes in wireless networks with contention-based medium access. The basic idea is to regard the process of contention control as carrying out a distributed strategy update algorithm to achieve the equilibrium of 
random access game. Hence the equilibrium (or steady state) and dynamic properties of a MAC can be understood or designed through the specification of the underlying random access game.

Specifically, in random access games, a wireless node's strategy is its channel access probability, and its payoff function includes both utility gain from channel access and cost from packet collision. Through the specification of per-node utility function, we can model a large class of system-wide quality-of-service (QoS) models, similar to that in utility framework for network flows [3]. We characterize the Nash equilibrium of random access games, study their dynamics, and propose algorithms (strategy evolutions) to achieve the Nash equilibrium. We show that system-wide fairness or service differentiation can be achieved in a distributed manner as long as each node executes a contention resolution algorithm that is designed to achieve the Nash equilibrium. We thus propose a novel medium access method derived from carrier sense multiple access/collision avoidance (CSMA/CA) in which each node estimates its conditional collision probability and adjusts its channel access probability accordingly. Our method adapts to continuous feedback signal (conditional collision probability) rather than binary contention signal, and each node tries to keep a fixed channel access probability (persistence probability or equivalently contention window size) specified by the Nash equilibrium of random access game. In addition to controllable performance objectives via the specification of per-node utility functions, as wireless nodes can estimate conditional collision probabilities by observing consecutive idle slots between transmissions, our access method can decouple contention control from handling failed transmissions. As a case study of medium access control design in the proposed game-theoretic framework, we present a concrete medium access method and show that it achieves optimal throughput, low collision, and good short-term fairness and can provide flexible service differentiations among wireless nodes.

Game-theoretic approach has recently been applied to network design and control. There are two complementary perspectives to the game-theoretic approach: economic perspective and engineering perspective. The economic perspective assumes network components or users are selfish and try to thwart selfish behaviors or induce cooperation using external mechanisms such as pricing. The engineering perspective envisions a scenario where network components or users are willing to cooperate but only have limited information about network states due to various practical constraints in real networks. In such a situation, the best an agent can do is to optimize some local or private objective and adjust its action based on limited information about the network state. We use noncooperative game to "model" such a situation (especially to capture the information structure of the system) and let network agents behave "selfishly" according to the game that is designed to guide individual agents to seek an equilibrium achieving some system-wide performance objective. Thus, from the engineering perspective, the focus is not on incentive issues, but on the implementation in practical networks. There are some controversies around the engineering perspective. Nonetheless, game-theoretic technique and language provide a powerful framework to reason about the engineering systems and guide their design and control. In this paper, we take the engineering perspective and implement distributed strategy update algorithms derived from the random access game as prescriptive control approach for channel access. ${ }^{1}$

\section{RELATED WORK}

Game-theoretic approach has been applied extensively to study random access (see, e.g., [4]-[10]). The work closest to ours is Jin et al. [4], which studies noncooperative equilibrium of Aloha networks and their local convergence, and Altman et al. [6] and Borkar et al. [7], which study distributed scheme for adapting random access. Our motivation, model, and results are different from those works. Our random access game model is intended as an analytical framework to reverse-engineer contention control as well as to guide the design of new medium access methods to achieve system-wide performance objectives. The specific structure of random access game is derived from a control-theoretic viewpoint of contention control, and the utility functions are derived from the steady operating points of existing protocols or the desired operating points we want medium access control to achieve. Inaltekin et al. [10] analyze Nash equilibria in multiple access with selfish nodes. Cagalj et al. [8] study selfish behavior in CSMA/CA networks and propose a distributed protocol to guide multiple selfish nodes to a Pareto-optimal Nash equilibrium. We do not consider such selfish behaviors of wireless users that tamper with wireless interfaces to increase their share of channel access as in [8]. In contrast, we use game-theoretic model to capture the information and implementation constraints encountered in real networks and design games to guide distributed users to achieve system-wide performance objectives.

There are lots of works on various enhancements and improvements to $802.11 \mathrm{DCF}$. We will only briefly discuss some designs that propose better contention resolution algorithms and that improve throughput by tuning the contention window according to the number of contending nodes. Aad et al. [11] introduce a slow decrease method to improve efficiency and fairness. Kwon et al. [12] propose a fast collision resolution algorithm for throughput improvement. Our design is different in terms of both contention measure and contention resolution algorithm. Bianchi et al. [13] and Cali et al. [14] propose to choose and compute an optimal contention window to maximize the throughput. They need sophisticated methods to estimate the number of contending nodes in the system, while our method does not require that information. There also exists extensive work on 802.11 QoS provisioning (see, e.g., [15]). Our access method can provide more flexible service differentiations through the specification of per-node utility functions, except for manipulating the length of interframe space.

Related work also includes [16] and [17]. Heusse et al. [16] propose a novel idle sense access method for a single-cell wireless LAN, which compares the mean number of idle slots between transmission attempts to the optimal value and adopts an additive increase and multiplicative decrease algorithm to dynamically control the contention window in order to improve

\footnotetext{
${ }^{1}$ However, our random access game model can also serve as a descriptive model of existing contention control protocols with selfish wireless nodes. This part is consistent with the economic perspective.
} 
throughput and short-term fairness. In our access method, wireless nodes estimate conditional collision probabilities by observing consecutive idle slots between transmissions. Therefore, like idle sense access method, our access method can decouple contention control from handling failed transmissions. Hu et al. [17] use a similar idea to propose a novel contention control method to maximize the bandwidth utilization and achieve proportional bandwidth allocation. This method observes two reference variables, the number of consecutive idle slots and the number of collisions between two consecutive successful transmissions, based on which to control the dequeueing rate of wireless nodes instead of dynamically tuning their contention windows. Our access method dynamically adjusts the contention window and achieves higher throughput (almost no throughput degradation compared to the optimal throughput) and provides a better guarantee of service differentiation.

A comparison with TCP congestion control is also in place. Contention control has a striking similarity with congestion control. They need to handle almost the same issues such as congestion/contention measure, load control (e.g., window update) algorithm, and decoupling load control from handling failed transmissions, etc. However, the interaction among wireless nodes is different from that among TCP flows, which means a different framework is needed to study contention control. Actually, one of the motivations of this work is to try to develop a parallel story for contention control to what has been done for TCP congestion control in the utility maximization framework (see, e.g., [3]).

\section{RANDOM ACCESS GAME}

Consider a set $N$ of wireless nodes in a wireless LAN with contention-based medium access. Associated with each wireless node $i \in N$ is its channel access probability $p_{i}(t)^{2}$ and a certain contention measure $q_{i}(t) \geq 0$ it can observe at time $t$. Node $i$ can observe its own access probability $p_{i}(t)$ and contention measure $q_{i}(t)$, but not those of other nodes. It adjusts its channel access probability $p_{i}(t)$ based only on $p_{i}(t)$ and $q_{i}(t)$

$$
p_{i}(t+1)=\mathcal{F}_{i}\left(p_{i}(t), q_{i}(t)\right) .
$$

The contention measure $q_{i}(t)$ depends on the channel access probabilities $\mathbf{p}(t)=\left(p_{i}(t), i \in N\right)$ chosen by the wireless nodes

$$
q_{i}(t)=\mathcal{G}_{i}(\mathbf{p}(t))
$$

Here, $\mathcal{F}_{i}$ models the contention resolution algorithms, and $\mathcal{G}_{i}$ models the contention measure update mechanisms. For convenience, we will also denote $\mathcal{G}_{i}(\mathbf{p})$ by $q_{i}(\mathbf{p})$.

We assume that (1) and (2) have an equilibrium (p, q). The fixed point of (1) defines an implicit relation between the equilibrium channel access probability $p_{i}$ and contention measure $q_{i}$

$$
p_{i}=\mathcal{F}_{i}\left(p_{i}, q_{i}\right) .
$$

2In our description of contention control, each wireless node's control variable is its channel access probability. For the same variable, we may have different ways to implement it in practical networks. Window-based control is seen as a way of "implementing" channel access probability.
Assume $\mathcal{F}_{i}$ is continuously differentiable and $\partial \mathcal{F}_{i} / \partial q_{i} \neq 0$ in $[0,1]$. Then, by the implicit function theorem [18], there exists a unique continuously differentiable function $F_{i}$ such that

$$
q_{i}=F_{i}\left(p_{i}\right) .
$$

Define the utility function of each node $i$ as

$$
U_{i}\left(p_{i}\right)=\int F_{i}\left(p_{i}\right) d p_{i} .
$$

Being an integral, and since $F_{i}\left(p_{i}\right)=q_{i} \geq 0, U_{i}(\cdot)$ is a continuous and nondecreasing function. It is reasonable to assume that $F_{i}(\cdot)$ is a decreasing function: The larger the contention, the smaller the channel access probability. This implies that $U_{i}(\cdot)$ is strictly concave.

With the above utility function, we define a random access game as follows.

Definition 1: A random access game $\mathcal{G}$ is defined as a triple $\mathcal{G}:=\left\{N,\left(S_{i}\right)_{i \in N},\left(u_{i}\right)_{i \in N}\right\}$, where $N$ is a set of players (wireless nodes), player $i \in N$ strategy $S_{i}:=\left\{p_{i} \mid p_{i} \in\left[\nu_{i}, \omega_{i}\right]\right\}$ with $0 \leq \nu_{i}<\omega_{i}<1$, and payoff function $u_{i}(\mathbf{p}):=U_{i}\left(p_{i}\right)-p_{i} q_{i}(\mathbf{p})$ with utility function $U_{i}\left(p_{i}\right)$ and contention measure $q_{i}(\mathbf{p})$.

We constrain the strategy $p_{i} \in S_{i}$ to be strictly less than 1 in order to prevent a node from exclusively occupying wireless channel. Note that the throughput of node $i$ is proportional to $p_{i}$ if there is no collision, and $q_{i}$ can be seen as contention price for node $i$. Thus, the payoff function $u_{i}(\cdot)$ has a nice economic interpretation: the net gain of utility from channel access, discounted by contention cost. The key to understanding contention control is to regard (1)-(2) as the strategy update algorithm to achieve the equilibrium of the random access game. Hence, the equilibrium (or steady-state) properties of a contention control protocol can be understood and designed through the specification of the utility function $U_{i}\left(p_{i}\right)$ and the contention measure $q_{i}$ (e.g., collision probability). Their specification defines the underlying random access game whose equilibrium determines the steady-state properties such as throughput, fairness, and collision of the contention control protocol. The adaptation of channel access probability can be specified through $(\mathcal{F}, \mathcal{G})$ and corresponds to different strategies to approach the equilibrium of the game.

Random access game $\mathcal{G}$ is defined in a rather general manner. As we saw above, it can be reverse engineered from given medium access control methods. Regarding the design of medium access control (i.e., forward-engineering), we can choose to implement any contention measures and specify any utility functions we think appropriate. To make our presentation and theoretical development concrete, in this paper we choose conditional collision probability as contention measure, i.e.,

$$
q_{i}(\mathbf{p}):=1-\prod_{j \in I_{i}}\left(1-p_{j}\right), \quad i \in N
$$

where $I_{i}$ denotes the set of nodes that interfere with the transmission of node $i$. As will become clear later, such a choice of contention measure has two nice properties, among others. First, conditional collision probability is an accurate measure of contention in the network. Each measurement of conditional collision probability provides multibit information of the contention. This makes it easier for an equation-based contention resolution 
algorithm to stabilize the network into a steady state with a target fairness (or service differentiation) and high efficiency. Second, wireless nodes can estimate conditional collision probabilities without using explicit feedback, which enables the decoupling of contention control from handling failed transmissions.

In the rest of this section and Section IV, we focus on singlecell wireless LANs, ${ }^{3}$ and will consider multicell wireless LANs in Section V. Before proceeding, we summarize the assumptions that will be used in this paper as follows.

A0: The utility function $U_{i}(\cdot)$ is continuously differentiable, strictly concave, and with finite curvatures that are bounded away from zero, i.e., there exist some constants $\mu$ and $\lambda$ such that $1 / \mu \geq-1 / U_{i}^{\prime \prime}\left(p_{i}\right) \geq 1 / \lambda>0$.

A1: Let $\gamma(\mathbf{p})=\prod_{i \in N}\left(1-p_{i}\right)$ and denote the smallest eigenvalue of $\nabla^{2} \gamma(\mathbf{p})$ over $\mathbf{p}$ by $\nu_{\min }$. Then, $-\mu-\nu_{\min }<$ 0 .

A2: Functions $\Gamma_{i}\left(p_{i}\right):=\left(1-p_{i}\right)\left(1-U_{i}^{\prime}\left(p_{i}\right)\right), i \in N$ are all strictly increasing or all strictly decreasing.

Assumption A0 is a standard assumption in economics and can also be seen as derived from those assumptions on function $F_{i}(\cdot)$. A1 guarantees the uniqueness of the Nash equilibrium of the random access game. A2 guarantees the uniqueness of nontrivial Nash equilibrium, which we will define later.

\section{A. Nash Equilibrium}

We now analyze the equilibrium of random access game. The solution concept we use is the Nash equilibrium [19]. Denote the strategy (channel access probability) selection for all nodes but $i$ by $\mathbf{p}_{-i}:=\left(p_{1}, p_{2}, \ldots, p_{i-1}, p_{i+1}, \ldots, p_{|N|}\right)$ and write $\left(p_{i}, \mathbf{p}_{-i}\right)$ for the strategy profile $\left(p_{1}, p_{2}, \ldots, p_{i-1}, p_{i}, p_{i+1}, \ldots, p_{|N|}\right)$. A vector of access probability $\mathbf{p}^{*}$ is a Nash equilibrium if, for all nodes $i \in N, u_{i}\left(p_{i}^{*}, \mathbf{p}_{-i}^{*}\right) \geq u_{i}\left(p_{i}, \mathbf{p}_{-i}^{*}\right)$ for all $p_{i} \in S_{i}$. We see that the Nash equilibrium is a set of strategies for which no player has an incentive to change unilaterally.

Theorem 2: Under assumption A0, there exists a Nash equilibrium for random access game $\mathcal{G}$.

Proof: Since the strategy spaces $S_{i}$ are compact convex sets, and the payoff functions $u_{i}$ are continuous and concave in $p_{i}$, there exists a Nash equilibrium [19].

Since payoff function $u_{i}(\cdot)$ is concave in $p_{i}$, at the Nash equilibrium, $p_{i}^{*}$ satisfies

$$
\left(U_{i}^{\prime}\left(p_{i}^{*}\right)-q_{i}\left(\mathbf{p}^{*}\right)\right)\left(p_{i}-p_{i}^{*}\right) \leq 0, \quad \forall p_{i} \in S_{i}
$$

Define function $V(\mathbf{p}):=\sum_{i \in N}\left(U_{i}\left(p_{i}\right)-p_{i}\right)-\prod_{i \in N}\left(1-p_{i}\right)$. It is easy to verify that (7) is an optimality condition for the following optimization problem [20]:

$$
\max _{\mathbf{p} \in S_{1} \times \cdots \times S_{|N|}} V(\mathbf{p})
$$

i.e., the Nash equilibria of the random access game are optimal points of the problem (8).

Theorem 3: Suppose additionally assumption A1 holds. Then, random access game $\mathcal{G}$ has a unique Nash equilibrium.

Proof: The Hessian of function $V(\mathbf{p})$ is written as

$$
\nabla^{2} V(\mathbf{p})=\operatorname{diag}\left(\left[U_{1}^{\prime \prime}\left(p_{1}\right), \ldots, U_{|N|}^{\prime \prime}\left(p_{|N|}\right)\right]\right)-\nabla^{2} \gamma(\mathbf{p})
$$

\footnotetext{
${ }^{3}$ Single-cell means that every wireless node can hear every other node in the network-for example, a single-cell wireless access network.
}

Note that

$$
\operatorname{diag}\left(\left[U_{1}^{\prime \prime}\left(p_{1}\right), \ldots, U_{|N|}^{\prime \prime}\left(p_{|N|}\right)\right]\right) \preceq-\mu \mathbf{I}
$$

and

$$
\nabla^{2} \gamma(\mathbf{p}) \succeq \nu_{\min } \mathbf{I}
$$

Thus, under assumption A1

$$
\nabla^{2} V(\mathbf{p}) \preceq-\left(\mu+\nu_{\min }\right) \mathbf{I} \prec 0 .
$$

By second-order conditions [20], $V(\mathbf{p})$ is a strictly concave function over the strategy space. Therefore, the optimization problem (8) has a unique optimal, and hence the random access game has a unique Nash equilibrium.

The equilibrium condition (7) implies that, at the Nash equilibrium, $p_{i}^{*}$ either takes value at the boundaries of the strategy space $S_{i}$ or satisfies

$$
U_{i}^{\prime}\left(p_{i}^{*}\right)=q_{i}\left(\mathbf{p}^{*}\right)
$$

We call a Nash equilibrium $\mathbf{p}^{*}$ a nontrivial equilibrium if, for all nodes $i, p_{i}^{*}$ satisfies (10), and trivial equilibrium otherwise. In the remainder of this section, we will mainly focus on nontrivial Nash equilibria. It follows from (10) that at nontrivial Nash equilibrium

$$
\Gamma_{i}\left(p_{i}^{*}\right)=\gamma\left(\mathbf{p}^{*}\right)
$$

Note that the right-hand side of the above equation is independent of $i$. Thus, $\Gamma_{i}\left(p_{i}^{*}\right)=\Gamma_{j}\left(p_{j}^{*}\right)$ for any $i, j \in N$.

Theorem 4: Suppose assumption A2 holds. If the random access game $\mathcal{G}$ has a nontrivial Nash equilibrium, it must be unique.

Proof: Suppose that there are two nontrivial Nash equilibria $\overline{\mathbf{p}}$ and $\hat{\mathbf{p}}$. From (11), we require that there exist $\gamma_{1}, \gamma_{2}>0$ such that, for all $i$

$$
\begin{aligned}
& \Gamma_{i}\left(\bar{p}_{i}\right)=\gamma_{1} \\
& \Gamma_{i}\left(\hat{p}_{i}\right)=\gamma_{2}
\end{aligned}
$$

Since $\Gamma_{i}\left(p_{i}\right)$ is one-to-one, $\gamma_{1} \neq \gamma_{2}$. Without loss of generality, assume that $\Gamma_{i}\left(p_{i}\right)$ is increasing and $\gamma_{1}>\gamma_{2}$. Thus, $\bar{p}_{i}>\hat{p}_{i}$ for all $i$. By $(10), U_{i}^{\prime}\left(\bar{p}_{i}\right)=q_{i}(\overline{\mathbf{p}})>q_{i}(\hat{\mathbf{p}})=U_{i}^{\prime}\left(\hat{p}_{i}\right)$, which contradicts the fact that $U_{i}^{\prime}\left(p_{i}\right)$ is a decreasing function. Thus, random access game $\mathcal{G}$ has a unique nontrivial Nash equilibrium.

Theorem 4 complements Theorem 3. Although A1 guarantees the uniqueness of nontrivial Nash equilibrium when there is one, it only gives a sufficient condition for the uniqueness. When there exist nontrivial Nash equilibria while A1 does not hold, A2 guarantees the uniqueness of nontrivial equilibrium.

Each node $i$ can choose any utility function $U_{i}(\cdot)$ it thinks appropriate. If all nodes have the same utility functions, the system is said to have homogeneous users. If the nodes have different utility functions, the system is said to have heterogeneous users. The motivation for studying systems of heterogeneous users is to provide differentiated services to different wireless nodes. To this end, we further differentiate among symmetric and asymmetric equilibria as follows. 
Definition 5: A Nash equilibrium $\mathbf{p}^{*}$ is said to be a symmetric equilibrium if $p_{i}^{*}=p_{j}^{*}$ for all $i, j \in N$, and an asymmetric equilibrium otherwise.

For a system of homogeneous users, both symmetric and asymmetric Nash equilibria are possible. For example, take $U_{i}\left(p_{i}\right)=a \ln \left(a+p_{i}\right)$ with $a>0$ and $p_{i} \in[0,1)$. In addition to nontrivial symmetric Nash equilibrium $\mathbf{p}^{*}$ that satisfies $\frac{a}{a+p_{i}^{*}}=1-\left(1-p_{i}^{*}\right)^{|N|-1}, i \in N$, when the number of wireless nodes $|N|>2$ there exists a family of nontrivial asymmetric Nash equilibria $\mathbf{p}^{*}$ that satisfy $p_{i}^{*}=1-\left(\frac{1}{a+1}\right)^{\frac{1}{|N|-2}}$, $p_{j}^{*}=\frac{a\left(1-p_{i}^{*}\right)}{a+p_{i}^{*}}$ for all $j \in N$ and $j \neq i$.

Since by symmetry there must be multiple asymmetric Nash equilibria if there exists any, the following result follows directly from Theorems 3 and 4.

Corollary 6: For a system of homogeneous users, suppose assumption A1 or A2 holds. If the random access game has a nontrivial Nash equilibrium, it must be unique and symmetric. More generally, for a system with several classes of homogeneous users, under the same assumption, if the random access game has a nontrivial Nash equilibrium, it must be unique and symmetric. 45

Corollary 6 guarantees the uniqueness of nontrivial Nash equilibrium, and moreover, it guarantees fair sharing of wireless channel among the same class of wireless nodes and provides service differentiation among different classes of wireless nodes. This will facilitate the analysis of dynamic property of random access games and the design of medium access control.

Remark: Since some player takes a strategy at the boundary of the strategy space at trivial Nash equilibrium, a trivial Nash equilibrium usually has great unfairness or low payoff. Therefore, nontrivial Nash equilibrium is desired. If there does not exist any nontrivial Nash equilibrium for a random access game, we may need to look for an alternative solution other than the Nash equilibrium. For example, we may use Nash bargaining framework in cooperative game theory to derive a desired equilibrium solution.

\section{B. Dynamics of Random Access Game}

The dynamics of game studies how interacting players could converge to a Nash equilibrium. It is a difficult problem in general, as pointed out in [19] that "game theory lacks a general and convincing argument that a Nash outcome will occur." In the setting of random access, players (wireless nodes) can observe the outcome (e.g., packet collision or successful transmission) of the actions of others, but do not have direct knowledge of other player actions and payoffs. We consider repeated play of random access game and look for an update mechanism in which players repeatedly adjust strategies in response to observations of other player actions so as to achieve the Nash equilibrium.

The simplest strategy update mechanism is of best response sort: At each stage, every node chooses the best response to the

\footnotetext{
${ }^{4}$ For a system with several classes of users, a Nash equilibrium is symmetric if the users of the same class choose the same strategy at equilibrium.

${ }^{5}$ There are many ways to give conditions on the existence of nontrivial Nash equilibrium; see [21] for one example condition. Here, we do not do that since those conditions intended for the game with arbitrary utility functions are restrictive. It is more sensible to give the existence condition for a concrete game with concrete utility functions; see Section IV-A for an example.
}

actions of all the other nodes in the previous round. Mathematically, at stage $t+1$, node $i \in N$ chooses a channel access probability

$$
p_{i}(t+1)=G_{i}(\mathbf{p}(t)):=\arg \max _{p \in S_{i}}\left(U_{i}(p)-p q_{i}(\mathbf{p}(t))\right) .
$$

Clearly, if the above dynamics reach a steady state, then this state is a Nash equilibrium. Nonetheless, there are no convergence results for general games using these dynamics.

We thus consider an alternative strategy update mechanism called gradient play [22]. Compared to "best response" strategy, gradient play can be viewed as a "better response." In gradient play, every player adjusts a current channel access probability gradually in a gradient direction suggested by observations of other player actions. Mathematically, each node $i \in N$ updates its strategy according to

$$
p_{i}(t+1)=\left[p_{i}(t)+f_{i}\left(p_{i}(t)\right)\left(U_{i}^{\prime}\left(p_{i}(t)\right)-q_{i}(\mathbf{p}(t))\right)\right]^{S_{i}}
$$

where the stepsize $f_{i}(\cdot)>0$ can be a function of the strategy of player $i$ and " $S_{i}$ " denotes the projection onto the player $i$ strategy space. The gradient play admits a nice economic interpretation if we interpret the conditional collision probability $q_{i}$ as contention price for node $i$ : If the marginal utility $\frac{d U_{i}\left(p_{i}\right)}{d p_{i}}$ is greater than contention price, we increase the access probability, and if the marginal utility is less than contention price, we decrease the access probability. The following result is immediate.

Lemma 7: By the equilibrium condition (7), the Nash equilibria of random access game $\mathcal{G}$ are fixed points of the gradient play (13), and vice versa.

Theorem 8: Suppose assumptions A0 and A1 hold. The gradient play (13) converges to the unique Nash equilibrium of random access game $\mathcal{G}$ if, for any $i \in N$, the stepsize $f_{i}<$ $\frac{2}{\lambda+|N|-1}$.

Proof: Consider function $V(\mathbf{p}):=\sum_{i \in N}\left(U_{i}\left(p_{i}\right)-p_{i}\right)-$ $\prod_{i \in N}\left(1-p_{i}\right)$. Define a matrix $B(\mathbf{p}):=-\nabla^{2} V(\mathbf{p})$. We have

$$
\|B(\mathbf{p})\|_{2}^{2} \leq\|B(\mathbf{p})\|_{\infty} \cdot\|B(\mathbf{p})\|_{1} .
$$

Since $B(\mathbf{p})$ is symmetric, $\|B(\mathbf{p})\|_{\infty}=\|B(\mathbf{p})\|_{1}$, and hence

$$
\begin{aligned}
\|B(\mathbf{p})\|_{2} & \leq\|B(\mathbf{p})\|_{\infty}=\max _{i} \sum_{j}[B(\mathbf{p})]_{i j} \\
& =\max _{i} \sum_{j}\left(-U_{i}^{\prime \prime}\left(p_{i}\right) \delta_{i, j}+\left(1-\delta_{i, j}\right) \prod_{k \neq i, j}\left(1-p_{k}\right)\right) \\
& \leq \max _{i}\left\{-U_{i}^{\prime \prime}\left(p_{i}\right)\right\}+|N|-1 \\
& \leq \lambda+|N|-1 .
\end{aligned}
$$

By Taylor expansion, we have

$$
\begin{aligned}
& V(\mathbf{p}(t+1))-V(\mathbf{p}(t)) \\
& =\nabla V(\mathbf{p}(t)) \cdot(\mathbf{p}(t+1)-\mathbf{p}(t)) \\
& \quad+\frac{1}{2}(\mathbf{p}(t+1)-\mathbf{p}(t))^{T} \cdot \nabla^{2} V(\overline{\mathbf{p}}) \cdot(\mathbf{p}(t+1)-\mathbf{p}(t)) \\
& \geq \\
& \quad \nabla V(\mathbf{p}(t)) \cdot(\mathbf{p}(t+1)-\mathbf{p}(t)) \\
& \quad-\frac{\lambda+|N|-1}{2}\|\mathbf{p}(t+1)-\mathbf{p}(t)\|_{2}^{2}
\end{aligned}
$$

where $\overline{\mathbf{p}} \in\{\mathbf{p} \mid \mathbf{p}=a \mathbf{p}(t)+(1-a) \mathbf{p}(t+1), a \in[0,1]\}$. Now, by the projection theorem [20]

$$
\left(p_{i}(t)+f_{i} \nabla_{i} V(\mathbf{p}(t))-p_{i}(t+1)\right)\left(p_{i}(t)-p_{i}(t+1)\right) \leq 0
$$


from which we obtain

$$
\nabla_{i} V(\mathbf{p}(t))\left(p_{i}(t+1)-p_{i}(t)\right) \geq \frac{1}{f_{i}}\left(p_{i}(t+1)-p_{i}(t)\right)^{2} .
$$

Thus, we have

$$
\begin{aligned}
V(\mathbf{p}(t+1)) & -V(\mathbf{p}(t)) \\
\geq & \sum_{i}\left(\frac{1}{f_{i}}-\frac{(\lambda+|N|-1)}{2}\right)\left(p_{i}(t+1)-p_{i}(t)\right)^{2} .
\end{aligned}
$$

Thus, if $f_{i}\left(p_{i}\right)<\frac{2}{\lambda+|N|-1}, V(\mathbf{p}(t+1))-V(\mathbf{p}(t)) \geq 0$. We see that $V(\mathbf{p})$ will keep increasing until the system reaches a fixed point of (13). By Theorem 3 and Lemma 7, under assumptions $\mathrm{A} 0$ and $\mathrm{A} 1,(13)$ has a unique fixed point in strategy space. Hence, the gradient play (13) converges to the unique Nash equilibrium of random access game $\mathcal{G}$.

Theorem 8 guarantees the convergence of distributed gradient play to the Nash equilibrium. If a backoff mechanism is implemented, each node $i \in N$ updates its contention window $c w_{i}$ as follows:

$$
c w_{i}(t)=\frac{2-p_{i}(t)}{p_{i}(t)}
$$

Equation (14) follows from the relation $p=\frac{2}{c w+1}$ that relates channel access probability $p$ to a constant contention window $c w$. This relation can be derived under the decoupling approximation for a set of wireless nodes with constant contention windows (see, e.g., [23] and [24]). The decoupling approximation is an extremely accurate approximation, as validated by extensive simulations reported in, e.g., [23] and [24]

In practice, there will be estimation error in the contention measure. Then, the gradient play can be written as

$$
p_{i}(t+1)=p_{i}(t)+f\left(U_{i}^{\prime}\left(p_{i}(t)\right)-q_{i}(\mathbf{p}(t))-e_{i}(t)\right)
$$

where $e_{i}$ denotes the estimation error in node $i$ 's conditional collision probability. ${ }^{6}$ We assume that the estimation errors are bounded, i.e., there exists a constant $\varepsilon>0$ such that $\|\mathbf{e}(t)\|_{2} \leq$ $\varepsilon$. We will show that under certain conditions, the gradient play (15) with estimation error converges to a neighborhood of nontrivial Nash equilibrium, where the size of the neighborhood depends on the accuracy of the contention measure estimation.

Theorem 9: Let $\mathbf{p}^{*}$ be a nontrivial Nash equilibrium. Suppose that $\nabla^{2} V(\mathbf{p})$ is nonsingular. Then, there exist a $\delta$ and a unique continuously differentiable function $\psi: R^{|N|} \rightarrow R^{|N|}$ defined on a $\delta$-neighborhood of the origin such that

$$
U_{i}^{\prime}(\psi(\mathbf{e}))=q_{i}(\psi(\mathbf{e}))+e_{i}, i \in N .
$$

Proof: At nontrivial Nash equilibrium $\mathbf{p}^{*}$, we have

$$
U_{i}^{\prime}\left(\mathbf{p}^{*}\right)-q_{i}\left(\mathbf{p}^{*}\right)-0=0, i \in N .
$$

\footnotetext{
${ }^{6}$ For the simplicity of presentation, we choose the same stepsize for all wireless nodes and neglect the projection operation. However, similar results as those of Theorems 9 and 10 can be established for the general case with heterogeneous stepsizes and the projection operation. The key is to note that function $[\mathbf{p}+\mathbf{f} \cdot(\nabla V(\mathbf{p})-\mathbf{e})]^{S_{1} \times \cdots \times S_{|N|}}-\mathbf{p}$ is one-to-one for fixed $\mathbf{e}$, and then apply the implicit function theorem for nonsmooth functions.
}

If $\nabla^{2} V(\mathbf{p})$ is nonsingular, function $\nabla V(\mathbf{p})-\mathbf{e}=\left(U_{1}^{\prime}\left(p_{1}\right)-\right.$ $\left.q_{1}(\mathbf{p})-e_{1}, \ldots, U_{|N|}^{\prime}\left(p_{|N|}\right)-q_{|N|}(\mathbf{p})-e_{|N|}\right)^{T}$ satisfies the conditions of the implicit function theorem [18]. The result follows directly from the implicit function theorem.

Theorem 10: Assume the same conditions as in Theorem 8. For any $\epsilon>0$, there exists a $\varepsilon>0$ such that if $\|\mathbf{e}\|_{2}<\varepsilon$, then the gradient play (15) with estimation errors converges into an $\epsilon$-neighborhood of nontrivial Nash equilibrium.

Proof: By Theorem 9, for any $\epsilon>0$, there exists a $\delta$ such that $\|\mathbf{e}\|_{2}<\delta$ implies that $\left\|\psi(\mathbf{e})-\mathbf{p}^{*}\right\|_{2}<\epsilon-2 f \delta$.

Now, under the gradient play (15), we have

$$
\begin{aligned}
& V(\mathbf{p}(t+1))-V(\mathbf{p}(t)) \\
& \geq \sum_{i}\left(\frac{1}{f}-\frac{\lambda+|N|-1}{2}\right)\left(p_{i}(t+1)-p_{i}(t)\right)^{2} \\
& \quad+\sum_{i} f e_{i}(t)\left(p_{i}(t+1)-p_{i}(t)\right) \\
& \geq\left(\frac{1}{f}-\frac{\lambda+|N|-1}{2}\right)\left(\|\mathbf{p}(t+1)-\mathbf{p}(t)\|_{2}^{2}\right. \\
& \left.-\frac{f^{2}}{2\left(\frac{1}{f}-\frac{\lambda+|N|-1}{2}\right)^{2}}\|\mathbf{e}(t)\|_{2}^{2}\right) .
\end{aligned}
$$

If $\| \mathbf{p}(t+1)-\mathbf{p}(t)) \|_{2}>\frac{f^{2}}{\sqrt{2}(1-(\lambda+|N|-1) f / 2)} \varepsilon, V(\mathbf{p})$ will keep increasing. Therefore, the gradient play (15) will enter at least once into a region defined by $\|\nabla V(\mathbf{p})\|_{2} \leq$ $\frac{1}{\sqrt{2}(1-(\lambda+|N|-1) f / 2)} \varepsilon$. Take any $\varepsilon<\delta /(\sqrt{2}(1-(\lambda+|N|-$ 1) $f / 2)$ ), and we have $\|\nabla V(\mathbf{p})\|_{2}<\delta$, i.e., there exits a $\overline{\mathbf{e}}$ such that $\|\overline{\mathbf{e}}\|_{2}<\delta$ and $U_{i}^{\prime}\left(p_{i}\right)-q_{i}(\mathbf{p})-\bar{e}_{i}=0, i \in N$. It follows that the gradient play (15) will enter at least once into a neighborhood of $\mathbf{p}^{*}$ defined by $\left\|\mathbf{p}-\mathbf{p}^{*}\right\|_{2}=\left\|\psi(\overline{\mathbf{e}})-\mathbf{p}^{*}\right\|_{2}<\epsilon-2 f \delta$. Once it enters into this neighborhood, the gradient play will stay inside a larger neighborhood defined by $\left\|\mathbf{p}-\mathbf{p}^{*}\right\|_{2}<\epsilon$.

Theorem 10 is the robust verification of the gradient play to the estimation error. It holds even under weaker condition that only requires the estimation errors to eventually remain within a $\varepsilon$-neighborhood of the origin. Being a dynamic feedback-based protocol, contention control usually operates at a region where the equilibrium condition is approximately satisfied due to various practical factors or constraints. Theorem 10 guarantees that this region is in a small neighborhood of the equilibrium, and thus the performance of the medium access method derived from the random access game is determined by the equilibrium.

\section{Medium Access Control Design}

Our ultimate purpose for studying random access games is to design medium access method with better performance. Corollary 6 and Theorem 8 suggest that random access games provide a general analytical framework to model a large class of systemwide QoS models (mainly in terms of throughput) via the specification of per-node utility functions, and system-wide fairness or service differentiation can be achieved in a distributed manner as long as each node executes a contention resolution algorithm that is designed to achieve the nontrivial Nash equilibrium.

Based on this understanding of the equilibrium and dynamics of random access games, we propose a novel medium 
TABLE I

MEdium ACCess Method Via GRAdient Play

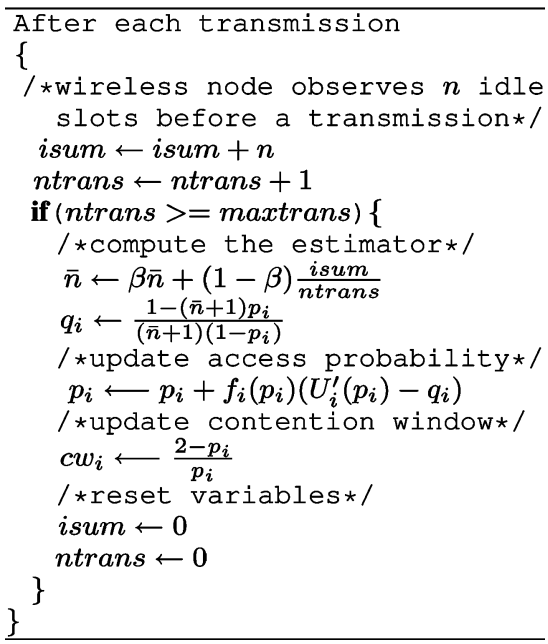

access method derived from CSMA/CA ${ }^{7}$ : instead of executing exponential backoff upon collisions, each node estimates its conditional collision probability and adjusts its channel access probability and contention window according to the gradient play (13)-(14); see Table I for a formal description (see Section IV-C for more explanation on notations). Our method adapts to continuous feedback signal (conditional collision probability) rather than binary feedback (packet collision) and stabilizes the network around a steady state specified by the Nash equilibrium of random access game. Therefore, it can achieve controllable performance objectives by choosing appropriate per-node utility functions. Our access method is an equation-based control, and its performance (such as throughput, collision and fairness) is determined by the Nash equilibrium. Note that $U_{i}^{\prime}\left(p_{i}(t)\right)-q_{i}(\mathbf{p}(t))$ specifies how far the current state is from the equilibrium. The contention window adjustment is small when the current state is close to the equilibrium, and large otherwise, independent of where the equilibrium is. This is in sharp contrast to the approach taken by 802.11 DCF, where window adjustment depends on just the current window size and is independent of where the current state is with respect to the target equilibrium. Thus, our access method can achieve better contention control and better short-term fairness.

Now, consider how wireless nodes can estimate conditional collision probabilities. Let $n$ denote the number of consecutive idle slots between two transmissions. Here, "a transmission" corresponds to a busy period in the channel when only a node transmits (i.e., a successful transmission) or multiple nodes transmit simultaneously (i.e., a collision). Since $n$ has the geometric distribution with parameter $\gamma(\mathbf{p})$, its mean $\bar{n}$ is given by $\bar{n}=\gamma(\mathbf{p}) /(1-\gamma(\mathbf{p}))$. Thus, each node can estimate its conditional collision probability by observing the average number of consecutive idle slots according to

$$
q_{i}=1-\frac{\gamma(\mathbf{p})}{1-p_{i}}=\frac{1-(\bar{n}+1) p_{i}}{(\bar{n}+1)\left(1-p_{i}\right)} .
$$

\footnotetext{
${ }^{7}$ We can also design medium access methods based on the ordinary Alohatype access method [25]. One of the main differences would be that, without CSMA, wireless nodes have to estimate the contention measure based on explicit feedback (i.e., failed transmissions).
}

With this estimation of the contention measure, our access method can decouple contention control from handling packet losses and is immune to those problems incurred in methods that infer channel contention from packet collisions.

In Section IV, we will study a concrete random access game and the corresponding medium access control design as a case study for the proposed design methodology in game-theotic framework. We will discuss more on the design of our medium access method.

\section{A CASE STUdy}

We have mostly ignored the design of utility functions in the previous section. In the following, we will derive utility functions and random access games from the desired operating points and design medium access methods accordingly.

Consider a single-cell wireless network with $L$ classes of users. Each class $l$ is associated with a weight $\phi_{l}$, and without loss of generality, we assume $\phi_{1}=\phi_{\max }>\phi_{2}>\cdots>\phi_{L}$. We want to achieve maximal throughput under the weighted fairness constraint

$$
\frac{\mathcal{T}_{l}}{\mathcal{T}_{m}}=\frac{\phi_{l}}{\phi_{m}}, 1 \leq l, m \leq L
$$

where $\mathcal{T}_{l}$ is the throughput of a class- $l$ node. Let $\zeta=\sum_{i \in N} p_{i}$. Under the assumption of the Poisson arrival process [25], the channel idle probability is approximately

$$
\prod_{i \in N}\left(1-p_{i}\right) \approx e^{-\zeta}
$$

and the aggregate successful packet transmission probability is $\zeta e^{-\zeta}$. Hence, the aggregate throughout can be written as

$$
\mathcal{T}=\frac{\zeta e^{-\zeta} P}{e^{-\zeta} \sigma+\zeta e^{-\zeta} T_{s}+\left(1-e^{-\zeta}-\zeta e^{-\zeta}\right) T_{c}}
$$

which achieves maximum at $\zeta^{*}$ that solves

$$
\left(1-\zeta^{*}\right) e^{\zeta^{*}}=1-\frac{\sigma}{T_{c}}
$$

where $P$ is the packet payload, $\sigma$ is the duration of an idle slot, and $T_{s}$ and $T_{c}$ are the durations the channel is sensed busy because of a successful transmission and during a collision, respectively.

Now, consider how to achieve the weighted fairness. When there is a large number of nodes accessing the channel, each user should sense approximately the same environment on average, and we can assume that each user has the same conditional collision probability. ${ }^{8}$ Thus, with equal packet payload sizes the throughput ratio between users of different classes is approximately the ratio between their channel access probabilities, i.e., we require

$$
\frac{p_{l}}{p_{m}}=\frac{\phi_{l}}{\phi_{m}}, 1 \leq l, m \leq L .
$$

The users of each class $l$ are associated with the same utility $U_{l}(\cdot)$ and thus the same function $\Gamma_{l}\left(p_{l}\right)=\left(1-p_{l}\right)\left(1-U_{l}^{\prime}\left(p_{l}\right)\right)$. Note that at the nontrivial Nash equilibrium, $\Gamma_{l}\left(p_{l}\right)=\gamma(\mathbf{p})$ for all $0 \leq l \leq L$. Thus, condition (21) can be achieved at a

\footnotetext{
${ }^{8}$ This assumption is similar to the decoupling approximation made in [23] and other works in performance analysis of 802.11 DCF.
} 
nontrivial Nash equilibrium if we design utility functions such that

$$
\Gamma_{l}\left(p_{l}\right)=h\left(\frac{p_{l}}{\phi_{l}}\right), 1 \leq l \leq L .
$$

By (19)-(20), a nontrivial equilibrium $p^{*}$ that achieves maximal throughput should satisfy

$$
h\left(\frac{p_{l}^{*}}{\phi_{l}}\right)=e^{-\zeta^{*}} .
$$

Equations (22)-(23) plus assumption A2 are the constraints on the desired utility functions that achieve the maximal throughput under the weighted fairness constraint at the nontrivial equilibrium. Note that when the number of wireless nodes is large, $p_{l}^{*}$ should be very small. A convenient choice that approximately satisfies the above constraints is

$$
h\left(\frac{p_{l}}{\phi_{l}}\right)=e^{-\zeta^{*}}\left(1+\frac{p_{l}}{\phi_{l}}\right)
$$

from which we derive a utility function

$$
U_{l}\left(p_{l}\right)=\left(1+\frac{e^{-\zeta^{*}}}{\phi_{l}}\right) p_{l}+e^{-\zeta^{*}}\left(1+\frac{1}{\phi_{l}}\right) \ln \left(1-p_{l}\right) .
$$

With this utility function, we define a random access game $\mathcal{G}_{1}$ where all players have the same strategy space $[0, \omega]$ with $\omega<1$ and different user classes are identified with different choices of the utility functions (25) with different weights.

Remark: The way we derive utility function (25) follows the same idea as in reverse-engineering (4)-(5): to derive the utility function and game from the equilibrium. To see this, note that the equilibrium condition (4) or equivalently (10) is encapsulated in (11). In the above utility function design, we derive function $\Gamma_{l}\left(p_{l}\right)$ from the desired equilibrium [see (24)] from which we derive the marginal utility $U_{l}^{\prime}\left(p_{l}\right)$ or equivalently $F_{l}\left(p_{l}\right)$. We then obtain the utility function by integration.

\section{A. Nash Equilibrium}

Now let us study the equilibrium of random access game $\mathcal{G}_{1}$.

Lemma 11: Suppose $\omega \geq \frac{1-e^{-\zeta^{*}}}{1+e^{-\zeta^{*}}\left\langle\phi_{\max }\right.}$. Then, random access game $\mathcal{G}_{1}$ has a unique nontrivial Nash equilibrium.

Proof: Suppose there are $n_{l}$ class- $l$ nodes, each with a channel access probability $p_{l}$. Let $c=e^{-\zeta^{*}}\left(1+\frac{p_{l}}{\phi_{l}}\right)$. Note that when $c=e^{-\zeta^{*}}, \prod_{l}\left(1-\phi_{l}\left(c e^{\zeta^{*}}-1\right)\right)^{n_{l}}>c$, and when $c=\min \left\{1, e^{-\zeta^{*}}\left(1+\frac{\omega}{\phi_{\max }}\right)\right\}, \prod_{l}\left(1-\phi_{l}\left(c e^{\zeta^{*}}-1\right)\right)^{n_{l}}<c$ if $\omega \geq \frac{1-e^{-\zeta^{*}}}{1+e^{-\zeta^{*}} / \phi_{\max }}$. Since $\prod_{l}\left(1-\phi_{l}\left(c e^{\zeta^{*}}-1\right)\right)^{n_{l}}$ and $c$ are continuous functions of $c$, there exists a solution $c^{*}$ to the following equilibrium equation [i.e., (11)]:

$$
\prod_{l}\left(1-\phi_{l}\left(c e^{\zeta^{*}}-1\right)\right)^{n_{l}}=c
$$

if $\omega \geq \frac{1-e^{-\zeta^{*}}}{1+e^{-\zeta^{*}} / \phi_{\max }}$. Thus, there exists a nontrivial Nash equilibrium $p_{l}=\phi_{l}\left(c^{*} e^{\zeta^{*}}-1\right)$ for random access game $\mathcal{G}_{1}$. Furthermore, $F_{l}\left(p_{l}\right)$ defined by (24) satisfies assumption A2. It follows from Theorem 4 that the nontrivial Nash equilibrium must be unique.
Theorem 12: Suppose additionally $\omega<1-\frac{e^{\zeta^{*}}}{1+1 / \phi_{\max }}$. Then, random access game $\mathcal{G}_{1}$ has a unique and nontrivial Nash equilibrium.

Proof: We have

$\operatorname{diag}\left(\left(U_{1}^{\prime \prime}\left(p_{1}\right), \ldots, U_{|N|}^{\prime \prime}\left(p_{|N|}\right)\right)\right) \preceq-e^{-\zeta^{*}}\left(1+\frac{1}{\phi_{\max }}\right) \mathbf{I}$ and

$$
\nabla^{2} \gamma(\mathbf{p})=\left(s^{T} s-\operatorname{diag}^{2}(s)\right) \prod_{i}\left(1-p_{i}\right)
$$

where $s=\left(\frac{1}{1-p_{1}}, \ldots, \frac{1}{1-p_{|N|}}\right)$. Note that $-\operatorname{diag}^{2}(s) \prod_{i}(1-$ $\left.p_{i}\right) \succeq \frac{-1}{1-\omega} \cdot s^{T} s$ is a positive semidefinite matrix of rank 1 , and thus $s^{T} s \prod_{i}\left(1-p_{i}\right) \succeq 0$. Therefore, if $\omega<1-\frac{e^{\zeta^{*}}}{1+1 / \phi_{\max }}$, $-\mu-\nu_{\min }<0$. It follows from Theorem 3 that random access game $\mathcal{G}_{1}$ has a unique Nash equilibrium. Since, by Lemma 11 , $\mathcal{G}_{1}$ has a nontrivial Nash equilibrium, the unique equilibrium must be nontrivial.

\section{B. Dynamics}

Assume that each node $i \in N$ adjusts its strategy according to gradient play

$$
\begin{aligned}
p_{i}(t+1) & =\left[p_{i}(t)+f_{i}\left(1-\frac{e^{-\zeta^{*}}\left(1+\frac{p_{i}}{\phi_{i}}\right)}{1-p_{i}}-q_{i}(\mathbf{p}(t))\right)\right]_{(28)}^{S_{i}} \\
c w_{i}(t) & =\frac{2-p_{i}(t)}{p_{i}(t)}
\end{aligned}
$$

where the stepsize $f_{i}>0$. Note that the Nash equilibria of random access game $\mathcal{G}_{1}$ are the fixed points of (28), and vice versa. The following result is immediate.

Theorem 13: Suppose $\frac{1-e^{-\zeta^{*}}}{1+e^{-\zeta^{*}} / \phi_{\max }} \leq \omega<1-\frac{e^{\zeta^{*}}}{1+1 / \phi_{\max }}$. Then, the system described by (28) converges to the unique and nontrivial Nash equilibrium of random access game $\mathcal{G}_{1}$ if, for any $i \in N$, the stepsize $f_{i}<\frac{2}{\lambda+|N|-1}$.

Proof: The result follows directly from Theorem 8 .

The condition $\frac{1-e^{-\zeta^{*}}}{1+e^{-\zeta^{*}} / \phi_{\max }} \leq \omega<1-\frac{e^{\zeta^{*}}}{1+1 / \phi_{\max }}$ is a mild assumption and admits a very large region in the parameter space. The Nash equilibrium can be easily calculated by numerically solving fixed point (10). If we consider a system of homogenous users, we choose the same utility function, i.e., the same weight $\phi_{i}$ for all users. If we want to provide differentiated services, we can choose larger value of $\phi_{i}$ for the users of a higher priority class. For example, in wireless access network, we can assign a large $\phi_{i}$ value to the access point, because usually downlink traffic is greater than the traffic of mobile nodes.

\section{Medium Access Control Design}

We design a medium access method according to channel access probability and contention window update mechanism (28)-(29) by modifying a CSMA/CA access method such as 802.11 DCF [2]. The basic access mechanism in DCF works as follows. A node wishing to transmit senses the channel for a period of time equal to the distributed interframe space (DIFS) to check if it is idle. If the channel is determined to be idle, the node starts to transmit a DATA frame. If the channel is considered to be busy, the node waits for a random backoff time $b$, 
an integer uniformly distributed in the window $[0, c w-1]$ before attempting to transmit. Upon successful reception of the DATA frame, the receiving node waits for a short interframe space (SIFS) interval and then sends an ACK frame. When the node detects a failed transmission, it doubles the contention window $c w$ (exponential backoff). In order to avoid channel capture, a node must wait for a random backoff time, in the same way as if the channel is sensed busy, between two consecutive new packet transmissions. Note that DCF employs a discrete-time backoff scale. The time immediately following an idle DIFS is slotted with slot-time size $\sigma$. The backoff time counter is decremented as long as the channel is sensed idle during a time slot, and frozen when the channel is sensed busy, and reactivated when the channel is sensed idle again for a DIFS. The node transmits when the backoff time counter reaches zero. See [2] for more details.

As described in Section III-C, our medium access method makes two key modifications to 802.11 DCF. Instead of adjusting contention window $c w_{i}$ to a binary feedback signal (packet loss or successful transmission) and using exponential backoff algorithm, each node $i$ estimates its conditional collision probability $q_{i}$, which is a continuous feedback, and adjusts $c w_{i}$ according to algorithm (28)-(29).

There are several parameters in our medium access method. The parameter $\zeta^{*}$ depends only on the protocol parameters such as the duration of an idle slot that are specified by the system designer. The maximal channel access probability $\omega$ affects the number of the equilibria of random access game $\mathcal{G}_{1}$ but not the location of the equilibrium (i.e., the equilibrium properties of our design) once it satisfies the condition specified in Section IV-A. The weight $\phi_{l}$ determines the fairness or service differentiation. The system designer will specify a set of weights, according to the levels of quality of services he wants to provide, and each wireless node will choose a weight that corresponds to the specific level of service it desires. The parameters $f_{i}$ and maxtrans determine the dynamic properties such as stability and responsiveness. The stepsize $f_{i}$ affects the convergence speed. In practice, we will choose a constant stepsize for all nodes. The number of transmissions, maxtrans, for each node before updating its channel access probability and contention window, affects the convergence speed and the accuracy of the conditional collision probability estimation. Note that in strategy update algorithm (28)-(29), $t$ is not real time, but represents the stages at which the random access game is played. In our design, each node repeatedly plays game $\mathcal{G}_{1}$ every maxtrans transmissions, and the channel access probability and contention window are fixed between consecutive plays. If maxtrans is too large, it will take a longer time to reach the Nash equilibrium, but if maxtrans is too small, it will result in large estimation error in the average number of consecutive idle slots between transmissions and thus conditional collision probability. In order to achieve a good tradeoff between convergence speed and estimation accuracy, we will choose a relatively small value for maxtrans and estimate average number of consecutive idle slots between transmissions using an exponential weighted running average

$$
\bar{n} \longleftarrow-\beta \bar{n}+(1-\beta) \frac{\text { isum }}{\text { ntrans }}
$$

where $\beta \in[0,1)$. If $\beta$ is small, we weight history less, and if $\beta$ is large, we weight history more. The running average works as
TABLE II

PARAMETERS USED TO OBTAIN NUMERICAL RESULTS

\begin{tabular}{|c||c|}
\hline Slot Time $(\sigma)$ & $20 \mu s$ \\
\hline SIFS & $10 \mu s$ \\
\hline DIFS & $50 \mu s$ \\
\hline Basic Rate (br) & 11 Mbps \\
\hline Data Rate (dr) & $1 \mu s$ \\
\hline Propagation Delay $(\delta)$ & 192 bits \\
\hline PHY Header (ph) & 272 bits \\
\hline MAC Header (mh) & 112 bits \\
\hline ACK & 12000 bits \\
\hline Packet Payload (P)
\end{tabular}

a low-pass filter, and by choosing appropriate $\beta$ value, it gives better estimate than the "naive" estimator isum/ntrans.

By our access method, the system is designed to reach and operate around the Nash equilibrium of random access game $\mathcal{G}_{1}$. Thus, its performance is determined by the Nash equilibrium of $\mathcal{G}_{1}$. Consider a system of greedy nodes that always have packets to transmit. Denote the equilibrium channel access probability of node $i$ by $p_{i}$; we can calculate its throughput $\mathcal{T}_{i}$ and conditional collision probability $q_{i}$ as follows:

$$
\begin{aligned}
\mathcal{T}_{i} & =\frac{p_{i}\left(1-q_{i}\right) P}{\gamma \sigma+\sum_{i} p_{i}\left(1-q_{i}\right) T_{s}+\left(1-\gamma-\sum_{i} p_{i}\left(1-q_{i}\right)\right) T_{c}} \\
q_{i} & =1-\prod_{j \neq i}\left(1-p_{j}\right)
\end{aligned}
$$

where idle probability $\gamma=\gamma(\mathbf{p})=\prod_{i}\left(1-p_{i}\right)$ and

$$
\begin{aligned}
& T_{s}=\frac{p h}{b r}+\frac{m h+P}{d r}+S I F S+\frac{p h}{b r}+\frac{A C K}{d r}+D I F S+2 \delta \\
& T_{c}=\frac{p h}{b r}+\frac{m h+P}{d r}+D I F S+\delta .
\end{aligned}
$$

See Table II for other notations. Here, for simplicity, we have assumed an equal payload size. The throughput for general payload size distribution can calculated in a similar way [23], and the aggregate throughput is the summation of $\mathcal{T}_{i}$ over all nodes $i$.

\section{Performance}

We have conducted numerical experiments to evaluate the performance of our medium access method. We develop a packet-level simulator that implements our method and the standard 802.11 DCF basic access method (i.e., no RTS/CTS). The values for the parameters used to obtain numerical results are summarized in Table II. The system values are those specified in the $802.11 \mathrm{~b}$ standard with DSSS PHY layer [2]. Under these specifications, $\zeta^{*}=0.1625$ and $0.0811<\omega<0.4118$. We set $\omega=2 / 17$, which corresponds to a contention window size of 16. In all simulations, we set the following values of the control parameters: maxtrans $=10, f_{i}=0.025$, and $\beta=0.5$. The simulation results reported aim to zoom in on specific properties of our access method.

1) Throughput and Collision Overhead: We consider a network of homogeneous users with perfect channel (i.e., there is no corrupted frame that is due to channel error) and compare the throughput achieved by our access method and $802.11 \mathrm{~b}$ DCF as 

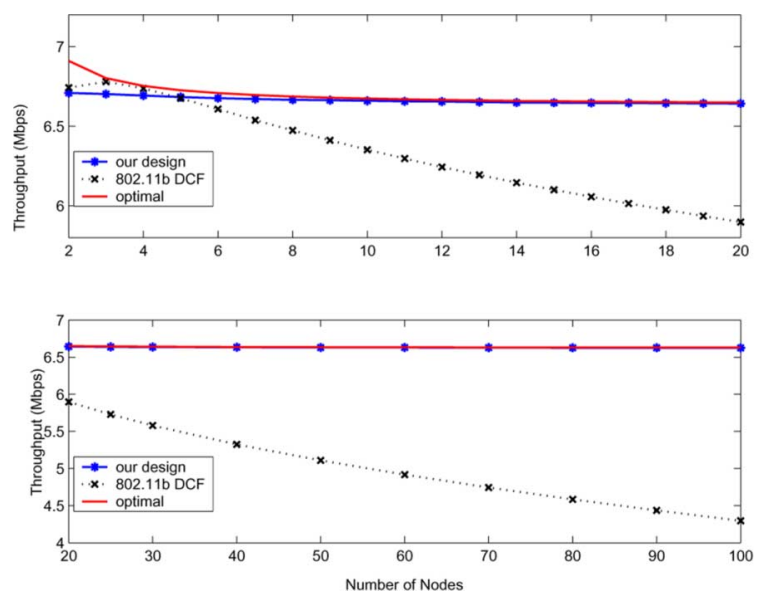

Fig. 1. Throughput comparison.

well as the maximal achievable throughput as our method is intended to achieve optimal throughput. In our design, each node $i$ has the same weight of 1 , i.e., $\phi_{i}=1$, since we consider homogenous users. In our numerical experiments with DCF, after a packet's $(m+1)$ th failed transmission, the contention window resets to the base contention window, where $m$ denotes the maximum backoff stage and is set to 5 for $802.11 \mathrm{~b}$. This is also equivalent to the packet being discarded after $m$ failed retransmissions.

Fig. 1 shows the aggregate throughput achieved by our design and DCF and the maximal achievable throughput. Our design almost achieves the optimal throughout, with only a slightly lower than optimal throughput for a network of a very small number of competing nodes. This also confirms that the approximations (19) and (24) made in deriving the utility functions (25) are very accurate approximations, and the contention measure estimator (18) and (30) give fairly accurate estimation of the conditional collision probability.

Compared to DCF, for a network of a very small number of wireless nodes, DCF provides a slightly higher throughput than our design. However, as the number of nodes increases, our access method achieves much higher throughput. With DCF, each new transmission starts with the base contention window and executes binary exponential backoff upon collisions, while, with our access method, nodes choose a constant contention window determined by the Nash equilibrium, which is "optimal" for the current contention level in the network. Thus, for a system of many competing nodes where the contention in the network is heavy, DCF will incur much more packet collisions than our access method, which results in much lower throughput, as shown in Fig. 1. This is further confirmed by the comparison of collision overhead between DCF and our access method, as shown in Fig. 2. Our access method achieves a very small, almost constant collision probability, better tradeoff between channel access and collision avoidance, and hence a higher throughput that is sustainable over a large range of numbers of competing nodes. Practically, this means that our access method can achieve higher throughput, but with fewer transmissions than DCF, which will benefit the whole system in many aspects such as lower energy usage and less interference to the wireless nodes of neighboring cells.

2) Fairness: It is well known that 802.11 DCF has a short-term fairness problem due to binary exponential backoff
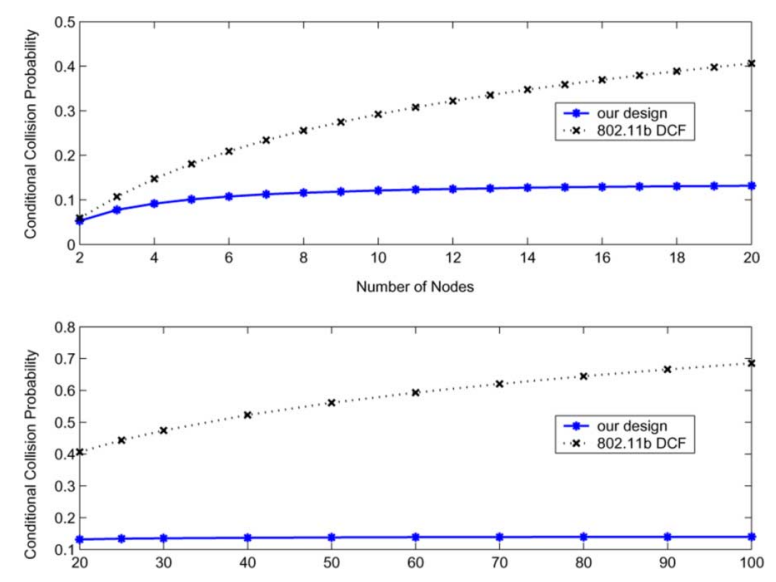

Fig. 2. Conditional collision probability comparison.

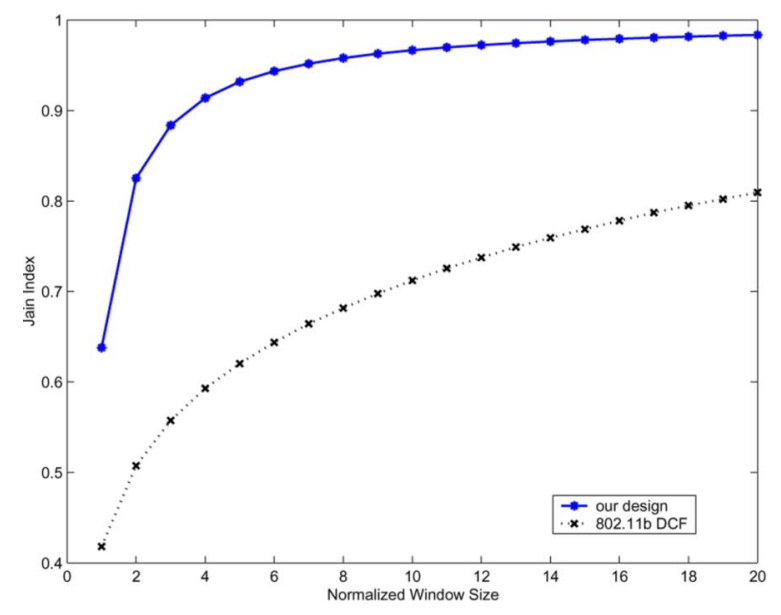

Fig. 3. Fairness comparison for a network of 40 competing nodes.

process. In our access method for a system of homogeneous users, wireless nodes have the same contention window size, specified by the symmetric Nash equilibrium of random access game $\mathcal{G}_{1}$. Thus, it is expected to have a better short-term fairness. Fig. 3 compares short-term fairness of our access method and DCF using Jain fairness index for the window sizes that are multiples of the number of wireless nodes [26]. Denote by $m_{i}$ the number of transmissions of node $i$ over a certain time window. Jain fairness index is defined by $\left(\sum_{i} m_{i}\right)^{2} /\left(|N| \sum_{i} m_{i}^{2}\right)$, and higher index means better fairness. We can see that our method provides much better short-term fairness than 802.11 DCF.

3) Dynamic Scenario: To evaluate the responsiveness and the convergence of our access method, we consider a dynamic scenario as follows. In the beginning, there are five greedy nodes in the network, which has converged to the equilibrium or the steady operating point. After 1004 more transmissions, five more nodes join in to compete for channel access. The five nodes then leave after 3000 transmissions. Fig. 4 shows the evolution of channel access probability and the corresponding contention window size of a long-stay node and a short-stay node, respectively. We see that our access method reaches the new equilibrium or steady operating point after only about totally 65 transmissions. That is about seven transmissions for each wireless node. The evolutions of different wireless nodes follows roughly the same trend. This is because they observe 

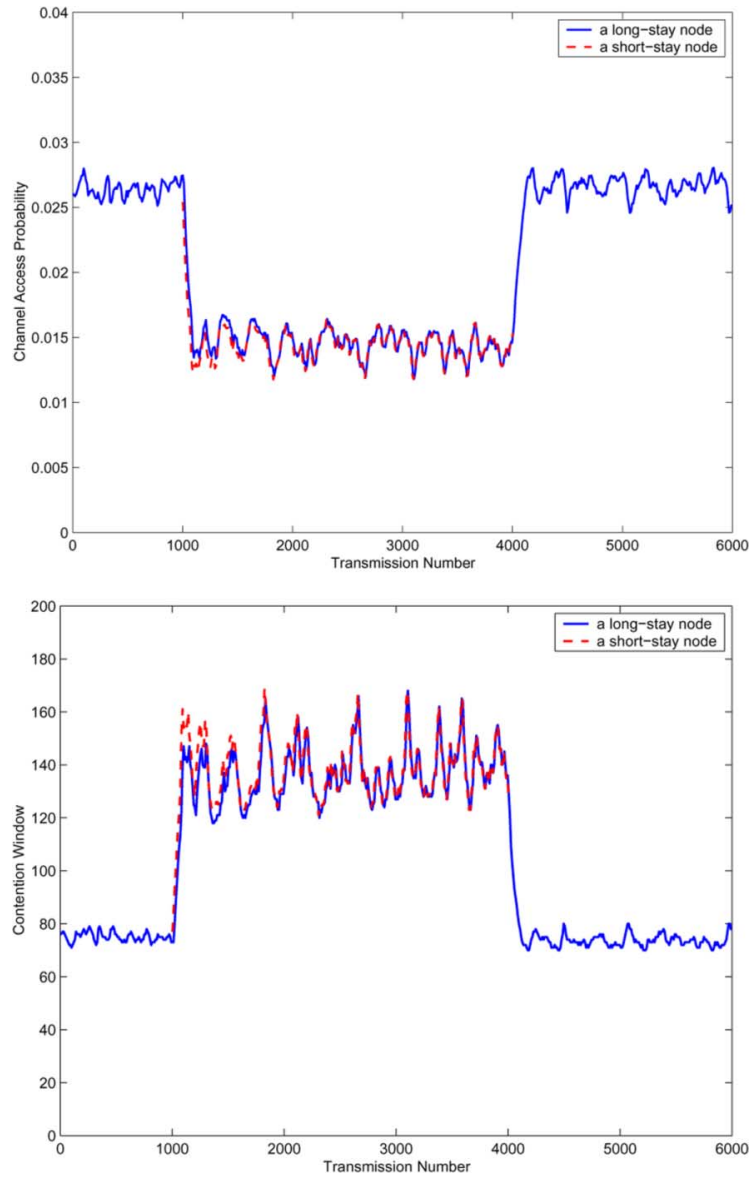

Fig. 4. Evolution of channel access probability and the corresponding contention window.

the same signal to estimate their contention measure, though the times when the long-stay node and the short-stay node update their channel access probabilities and contention windows are four transmissions away.

In order to respond quickly to the channel contention, in our access method when a wireless node $i$ enters the system or starts to have data to send after being idle, it will monitor the channel for a certain amount of time before transmitting to estimate current channel contention $q_{i}^{0}$. Based on this estimation, it will set its starting channel access probability to $p_{i}^{0}=\max \left\{\left(U_{i}^{\prime}\right)^{-1}\left(q_{i}^{0}\right), w\right\}$. In our simulation for the dynamic scenario, after entering the network, the short-stay nodes monitor the channel for the duration of three consecutive transmissions to estimate current channel contention to set the starting channel access probability and the corresponding contention window. This reflects in Fig. 4 where the starting channel access probability and contention window of the short-stay node are close to the channel access probability and contention window of the long-stay node.

4) Throughput Under Unreliable Channel: We now consider the network with unreliable channel, i.e., there exist corrupted frames due to channel error. Fig. 5 shows the aggregate throughput achieved by our design and $802.11 \mathrm{~b}$ DCF, with zero, $10 \%, 20 \%$, and $40 \%$ frame error rates, respectively. We see that, as expected, our contention resolution algorithm is insensitive
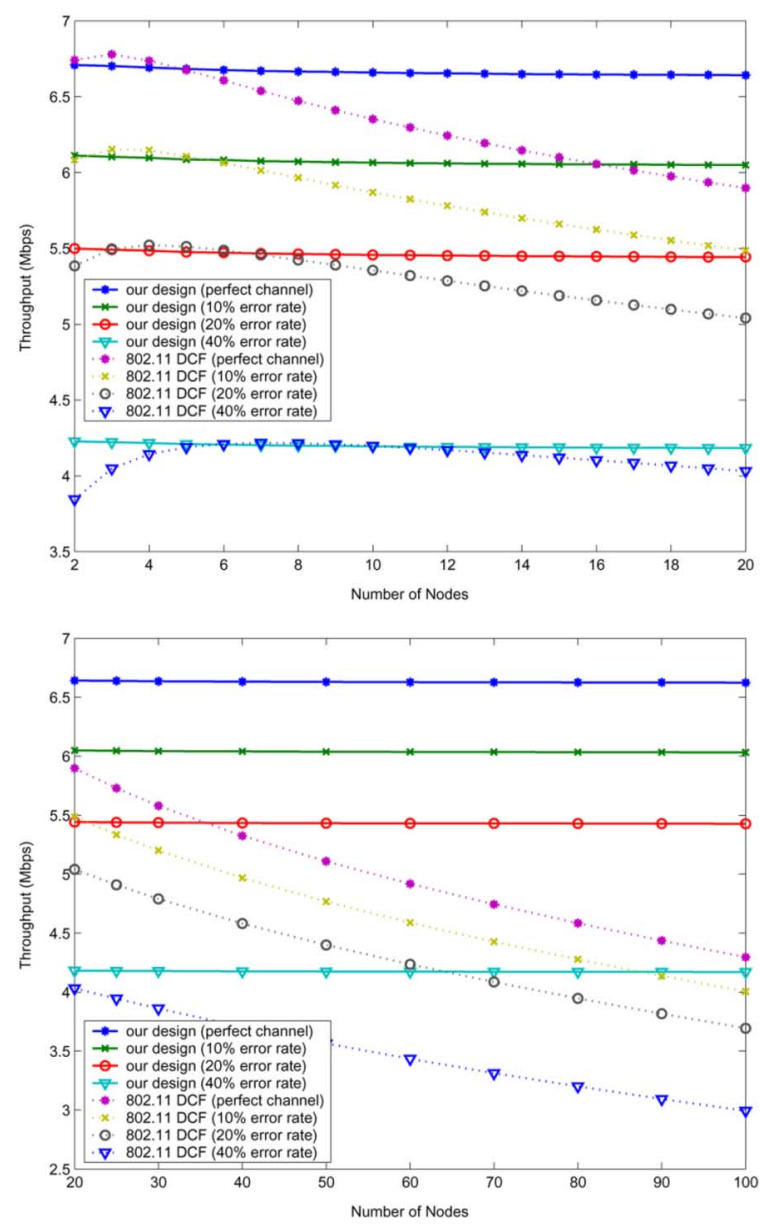

Fig. 5. Throughput comparison of our design and $802.11 \mathrm{~b}$ DCF with different frame error rates.

to channel error, and the throughput degradation comes solely from the corrupted frames due to channel error.

The impact of channel error on DCF is a bit "twisted." When the number of nodes is very small, the channel error leads to larger degradation in throughput. This is because packet collision is rare in this situation, and channel error results in more backoff and hence fewer transmissions. However, when the number of nodes is large, the channel error actually helps. This is because packet collision is frequent in this situation, and additional backoff due to the corrupted frames greatly reduces the chance of packet collision. Note that we have assumed an i.i.d channel error model. If the channel error comes in bursts, it will lead to greater throughput degradation of DCF and make DCF interact adversely with higher layer protocols such as TCP congestion control. Our access method has no such problems since its contention resolution algorithm is insensitive to the channel error.

5) Service Differentiation: As discussed before, we can provide service differentiation by choosing different utility functions for different classes of users. Regarding the concrete medium access method we consider, each node $i$ will receive different services by choosing different weights $\phi_{i}$. For the simplicity of presentation, we consider two classes of users. Assume that class 1 has $n_{1}$ users with weight $\phi_{1}=1$, corresponding to a higher priority of service, and class 2 has $n_{2}$ users with weight $\phi_{2}=0.5$, corresponding to a lower priority of 


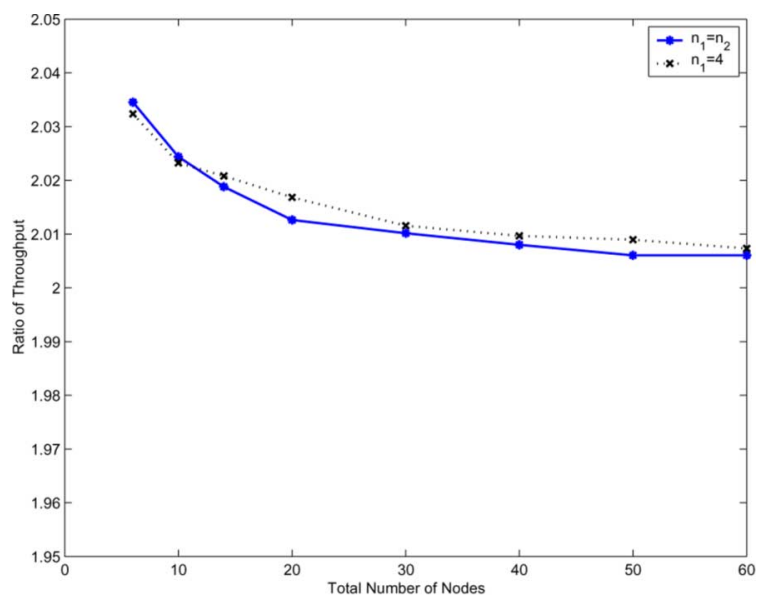

Fig. 6. The throughput ratio of a class -1 node with weight $\phi_{1}=1$ to a class -2 node with weight $\phi_{2}=0.5$.

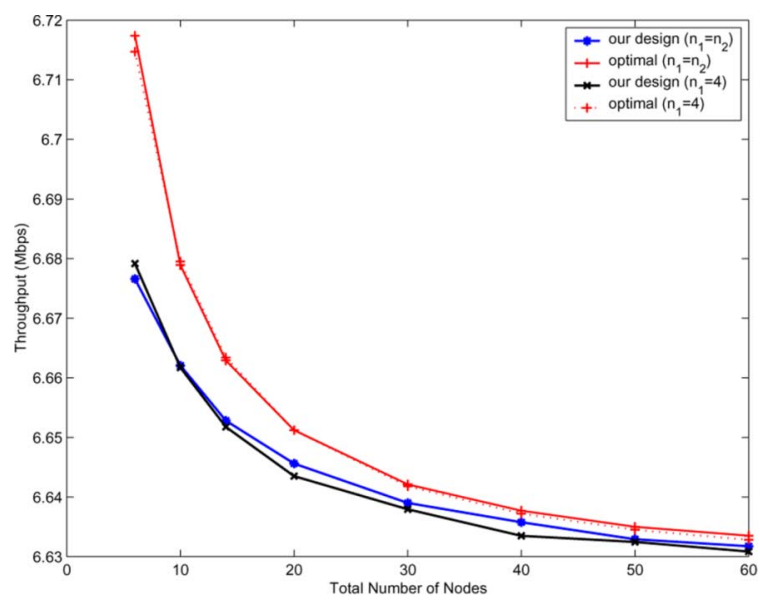

Fig. 7. The aggregate throughput versus the number of nodes.

service. Fig. 6 shows the throughput ratio of a class- 1 node to a class-2 node versus the total number of nodes for two different scenarios: two classes have equal number of users, and class 1 has fixed number of users. We see that the throughput ratio is almost exactly $\frac{\phi_{1}}{\phi_{2}}=2$. This also confirms that the condition (21) is a very accurate approximate requirement for achieving the desired service differentiation.

Fig. 7 shows the corresponding aggregate throughput by our access method. We see that our design almost achieves the maximal achievable throughput. This again confirms that the approximations (19) and (24) made in deriving the utility functions (25) are very accurate approximations.

\section{ExTENSION TO MUlTiCELl NetwORKS}

We now extend the above development to multicell wireless LANs in which some wireless nodes (e.g., those at the boundaries of the cells) may belong to more than one cell. Multicell networks are difficult to analyze because of the asymmetric information involved at different nodes. Mathematically, this reflects in the fact that the Jacobi matrix of the user payoff functions $\left(u_{1}(\mathbf{p}), \ldots, u_{|N|}(\mathbf{p})\right)^{T}$ is not a symmetric matrix. Nonetheless, we can still establish the uniqueness and convergence of the Nash equilibrium by generalizing the aforementioned conditions.

It is straightforward to verify that Theorem 2 holds for the multicell network, i.e., under assumption A0 there exists at least one Nash equilibrium for random access games $\mathcal{G}$. Let $J$ be the Jacobi matrix of the contention measure vector $\mathbf{q}(\mathbf{p})$, i.e., $J_{i j}=$ $\frac{\partial q_{i}(\mathbf{p})}{\partial p_{i}}$, and denote by $\nu_{J}$ the smallest eigenvalue of matrix $(J+$ $\left.J^{T}\right) / 2$ over $\mathbf{p}$.

Theorem 14: Suppose assumption A0 holds and $-\mu-\nu_{J}<$ 0 . Then, random access game $\mathcal{G}$ has a unique Nash equilibrium.

Proof: Since A0 holds, there exists at least one Nash equilibrium, denoted by $\mathbf{p}^{*}$. Define function $W(\mathbf{p}):=$ $\sum_{i \in N}\left(U_{i}^{\prime}\left(p_{i}\right)-U_{i}^{\prime}\left(p_{i}^{*}\right)\right)\left(p_{i}-p_{i}^{*}\right)-\sum_{i \in N}\left(q_{i}(\mathbf{p})-\right.$ $\left.q_{i}\left(\mathbf{p}^{*}\right)\right)\left(p_{i}-p_{i}^{*}\right)$. Note that $W\left(\mathbf{p}^{*}\right)=0$. Let $\bar{W}(\overline{\mathbf{p}})=$ $\sum_{i \in N}\left(U_{i}^{\prime}\left(\bar{p}_{i}\right)-q_{i}(\overline{\mathbf{p}})\right)\left(p_{i}-p_{i}^{*}\right)$. By Taylor expansion, we have

$$
\begin{aligned}
W(\mathbf{p})= & \bar{W}(\mathbf{p})-\bar{W}\left(\mathbf{p}^{*}\right) \\
= & \sum_{i \in N} U_{i}^{\prime \prime}\left(\hat{p}_{i}\right)\left(p_{i}-p_{i}^{*}\right)^{2}-\left(\mathbf{p}-\mathbf{p}^{*}\right) J(\hat{\mathbf{p}})\left(\mathbf{p}-\mathbf{p}^{*}\right)^{T} \\
= & \sum_{i \in N} U_{i}^{\prime \prime}\left(\hat{p}_{i}\right)\left(p_{i}-p_{i}^{*}\right)^{2}-\left(\mathbf{p}-\mathbf{p}^{*}\right) \\
& \times \frac{J(\hat{\mathbf{p}})+J^{T}(\hat{\mathbf{p}})}{2}\left(\mathbf{p}-\mathbf{p}^{*}\right)^{T} \\
\leq & -\left(\mu+\nu_{J}\right)\left\|\mathbf{p}-\mathbf{p}^{*}\right\|_{2}^{2}
\end{aligned}
$$

where $\hat{\mathbf{p}} \in\left\{\tilde{\mathbf{p}} \mid \tilde{\mathbf{p}}=a \mathbf{p}+(1-a) \mathbf{p}^{*}, a \in[0,1]\right\}$. If $-\mu-\nu_{J}<0$, $W(\mathbf{p})<0$ for any $\mathbf{p} \neq \mathbf{p}^{*}$. Now suppose there exists another Nash equilibrium, denoted by $\overline{\mathbf{p}}^{*}$, then $W\left(\overline{\mathbf{p}}^{*}\right)<0$. However, by equilibrium condition (7)

$$
\begin{aligned}
& W\left(\overline{\mathbf{p}}^{*}\right)= \sum_{i \in N}\left(U_{i}^{\prime}\left(\bar{p}_{i}^{*}\right)-q_{i}\left(\overline{\mathbf{p}}^{*}\right)\right)\left(\bar{p}_{i}^{*}-p_{i}^{*}\right) \\
&+\sum_{i \in N}\left(U_{i}^{\prime}\left(p_{i}^{*}\right)-q_{i}\left(\mathbf{p}^{*}\right)\right)\left(p_{i}^{*}-\bar{p}_{i}^{*}\right) \\
& \geq 0
\end{aligned}
$$

which contradicts the relation $W\left(\overline{\mathbf{p}}^{*}\right)<0$. Thus, random access game $\mathcal{G}$ has a unique Nash equilibrium.

The condition $-\mu-\nu_{J}<0$ is a generalization of assumption A1. As far as we know, our proof method for the uniqueness of the equilibrium is new. It only uses basic property of convexity, and is expected to provide a general technique to establish the uniqueness result.

Theorem 15: Assume the same conditions as in Theorem 14 and the same constant stepsize $f$ for the gradient play for all wireless nodes. The gradient play (13) converges to the unique Nash equilibrium of random access game $\mathcal{G}$ if the stepsize $f<$ $\frac{\mu+\nu_{J}}{\lambda^{2}+|N|(|N|-1)}$.

Proof: First note that the Nash equilibrium of random access game $\mathcal{G}$ is the fixed point of the gradient play (13), and vice versa. Define a mapping $\mathbf{M}(\mathbf{p})$

$$
M_{i}(\mathbf{p})=p_{i}+f_{i}\left(U_{i}^{\prime}\left(p_{i}\right)-q_{i}(\mathbf{p})\right) .
$$

The gradient play can be written as $p_{i}(t+1)=\left[M_{i}(\mathbf{p}(t))\right]^{S_{i}}$. Note that $\left\|\left[M_{i}(\mathbf{p})\right]^{S_{i}}-\left[M_{i}(\overline{\mathbf{p}})\right]^{S_{i}}\right\|_{2} \leq\left\|M_{i}(\mathbf{p})-M_{i}(\overline{\mathbf{p}})\right\|_{2}$. If we can show that $\mathbf{M}(\mathbf{p})$ is a contraction mapping, then the 
gradient play is a contraction mapping, too. Now, assume a constant stepsize $f$ for all nodes. For any two points $\mathbf{p}$ and $\overline{\mathbf{p}}$ in the strategy space, we have

$$
\begin{aligned}
&\|\mathbf{M}(\mathbf{p})-\mathbf{M}(\overline{\mathbf{p}})\|_{2}^{2} \\
& \leq\|\mathbf{p}-\overline{\mathbf{p}}\|_{2}^{2}+2 f \sum_{i \in N}\left(U_{i}^{\prime}\left(p_{i}\right)-U_{i}^{\prime}\left(\bar{p}_{i}\right)\right)\left(p_{i}-\bar{p}_{i}\right) \\
&-2 f \sum_{i \in N}\left(q_{i}(\mathbf{p})-q_{i}(\overline{\mathbf{p}})\right)\left(p_{i}-\bar{p}_{i}\right) \\
&+2 f^{2}\left(\sum_{i \in N}\left(U_{i}^{\prime}\left(p_{i}\right)-U_{i}^{\prime}\left(\bar{p}_{i}\right)\right)^{2}+\sum_{i \in N}\left(q_{i}(\mathbf{p})+q_{i}(\overline{\mathbf{p}})\right)^{2}\right) \\
& \leq\left(1-2 f\left(\mu+\nu_{J}\right)+2 f^{2} \lambda^{2}\right)\|\mathbf{p}-\overline{\mathbf{p}}\|_{2}^{2} \\
&+2 f^{2}(\mathbf{p}-\overline{\mathbf{p}}) J^{T}(\check{\mathbf{p}}) J(\hat{\mathbf{p}})(\mathbf{p}-\overline{\mathbf{p}})^{T} \\
& \leq\left(1-2 f\left(\mu+\nu_{J}\right)+2 f^{2} \lambda^{2}\right)\|\mathbf{p}-\overline{\mathbf{p}}\|_{2}^{2} \\
&+f^{2}(\mathbf{p}-\overline{\mathbf{p}})\left(J^{T}(\check{\mathbf{p}}) J(\hat{\mathbf{p}})+J^{T}(\hat{\mathbf{p}}) J(\check{\mathbf{p}})\right)(\mathbf{p}-\overline{\mathbf{p}})^{T} \\
& \leq\left(1-2 f\left(\mu+\nu_{J}\right)\right. \\
&\left.+2 f^{2} \lambda^{2}+2 f^{2}|N|(|N|-1)\right)\|\mathbf{p}-\overline{\mathbf{p}}\|_{2}^{2}
\end{aligned}
$$

where $\hat{\mathbf{p}}, \check{\mathbf{p}} \in\{\tilde{\mathbf{p}} \mid \tilde{\mathbf{p}}=a \mathbf{p}+(1-a) \overline{\mathbf{p}}, a \in[0,1]\}$, and in the third inequality we apply the same trick as in the proof of Theorem 14. We see that if the stepsize $f<\frac{\mu+\nu_{J}}{\lambda^{2}+|N|(|N|-1)}$, $\|\mathbf{M}(\mathbf{p})-\mathbf{M}(\overline{\mathbf{p}})\|_{2}^{2}<\|\mathbf{p}-\overline{\mathbf{p}}\|_{2}^{2}$. Thus $M(\mathbf{p})$ is a contraction mapping, and so is the gradient play. By the contraction mapping theorem [18], the gradient play will converge to the unique fixed point of (13), i.e., the unique Nash equilibrium of random access game $\mathcal{G}$.

The above condition on the stepsize looks strong, but seen from the proof, it is overly conservative. We are seeking other techniques to better bound the matrix norm to weaken this condition. If the medium access method designed according to the gradient play (13) of random access game $\mathcal{G}$ is used in multicell wireless LANs, Theorems 15 guarantees that it will operate around the equilibrium of random access game $\mathcal{G}$ and achieve the performance specified by the equilibrium.

\section{Some Comments on Reverse- ENGINEERING}

The dynamic model (1)-(2) of contention control is very general. The function $\mathcal{F}_{i}$ is usually a deterministic function, while the function $\mathcal{G}_{i}$ may be deterministic if the contention measure is a continuous variable or stochastic if the contention measure is a discrete variable. In the derivation of the utility function in Section III, we have implicitly assumed a continuous contention measure. If a discrete contention measure is used, the fixed point equation is usually not dictated directly by function $\mathcal{F}_{i}$ as in (3). Instead, the fixed point may be dictated by a certain function that relates the channel access probability $p_{i}$ and the average contention measure $q_{i}$

$$
p_{i}=F_{i}\left(p_{i}, q_{i}\right) .
$$

Starting with this fixed point equation and following the same procedure as in (4)-(5), we can define the utility function and a random access game and interpret the contention control protocol as a distributed strategy update algorithm to achieve the equilibrium of this game.

Take the IEEE 802.11 DCF as an example. DCF responds to whether there is a collision, and hence the measure of contention $q_{i}(t)$ in DCF is a binary feedback signal whose dependence on the channel access probability vector $\mathbf{p}(t)$ can be modeled by stochastic function $\mathcal{G}_{i}$

$$
\mathcal{G}_{i}(\mathbf{p})= \begin{cases}1, & \text { with probability } 1-\prod_{j \neq i}\left(1-p_{j}\right) \\ 0, & \text { with probability } \prod_{j \neq i}\left(1-p_{j}\right)\end{cases}
$$

It is well established that for a single-cell wireless LAN at steady state, the average channel access probability $p$ relates to the conditional collision probability $q=E\left[\mathcal{G}_{i}\right]$ as follows [23]:

$$
p=\frac{2(1-2 q)}{(1-2 q)(C W \min +1)+q C W \min \left(1-(2 q)^{m}\right)}
$$

where $C W$ min is the base contention window and $m$ is the maximum backoff stage. Following the procedure (4)-(5) to derive a utility function $U(p)$, we can define a random access game with payoff $U(p)-p q(\mathbf{p})$ and interpret DCF as a distributed strategy update algorithm to achieve the corresponding Nash equilibrium. Note that the dynamics of DCF cannot be described by (stochastic) gradient play. The convergence of DCF to the Nash equilibrium is guaranteed by the stability of the underlying Markov chain that describes the dynamics of DCF. However, we can design a medium access method according to the gradient play of this game, which will achieve the same throughput as that by DCF, but with better short-term fairness.

One of the key aspects of our reverse-engineering is to reverse-engineer the equilibrium (or steady operating point) of the contention control protocol, i.e., the equilibrium of the resulting game should recover the equilibrium (or steady operating point) of the contention control protocol; see (3) and (33). Our random access game framework applies to any dynamic contention-based medium access methods that have a steady operating point, such as the stabilized Aloha [25].

\section{CONCLUSION}

We have developed a game-theoretic model for contention control and propose a novel medium access method derived from CSMA/CA, in which each node estimates its conditional collision probability and adjusts its persistence probability or contention window according to a distributed strategy update mechanism achieving the Nash equilibrium. This results in controllable performance objectives through the specification of per-node utility functions. As wireless nodes can estimate conditional collision probabilities by observing consecutive idle slots between transmissions, we can decouple contention control from handling failed transmissions. As a case study of medium access control design in the proposed game-theoretic framework, we present a concrete medium access method that achieves optimal throughput, low collision, and good short-term fairness and can provide flexible service differentiations among wireless nodes. In addition to guiding medium access control design, the random access game model also provides an analytical framework to understand equilibrium properties and dynamic properties of different medium access protocols.

This paper has been focusing on laying out a theoretical framework. Much work remains to take it from a promising design framework to a full-fledged medium access control protocol. We are extending the framework to the network where wireless nodes have arbitrary traffic patterns (e.g., bursty packet arrival), which will be reported elsewhere. We will search for other contention resolution algorithms that could 
achieve Nash equilibria of random access games and other contention measures that admit robust estimation. We are also studying the issue of ensuring time fairness in a wireless network with rate diversity. We are also investigating the coexistence of our access method and 802.11 DCF that use different contention signals: how the resource is allocated to and shared among wireless nodes using different medium access methods. This issue is important for the deployment of the new access method. We also plan to implement our access method in a testbed to evaluate its performance against realistic physical characteristics of a network, especially to study the robust estimation of conditional collision probability and examine the setting of various control parameters that determine the dynamic properties of the access method.

\section{REFERENCES}

[1] T. Nandagopal, T. E. Kim, X. Gao, and V. Bharghhavan, "Achieving MAC layer fairness in wireless packet networks," in Proc. ACM MobiCom, 2000.

[2] Wireless LAN Media Access Control (MAC) and Physical Layer (PHY) Specifications, IEEE Standard 802.11, Jun. 1999.

[3] F. P. Kelly, A. K. Maulloo, and D. K. H. Tan, "Rate control for communication networks: Shadow prices, proportional fairness and stability," J. Oper. Res. Soc., vol. 49, no. 3, pp. 237-252, Mar. 1998.

[4] Y. Jin and G. Kesidis, "Equilibria of a noncooperative game for heterogeneous users of an Aloha networks," IEEE Commun. Lett., vol. 6, no. 7, pp. 282-284, Jul. 2002.

[5] A. B. MacKenzie and S. B. Wicker, "Stability of multipacket slotted Aloha with selfish users and perfect information," in Proc. IEEE INFOCOM, 2003, vol. 3, pp. 1583-1590.

[6] E. Altman, V. S. Borkar, and A. A. Kherani, "Optimal random access in networks with two-way traffic," in Proc. IEEE PIMRC, 2004, vol. 1, pp. 609-613.

[7] V. S. Borkar and A. A. Kherani, "Random access in wireless ad hoc networks as a distributed game," in Proc. WiOpt, 2004.

[8] M.Cagalj, S. Ganeriwal, I. Aad, and J. P. Hubaux, "On selfish behavior in CSMA/CA networks," in Proc. IEEE INFOCOM, 2005, vol. 4, pp. 2513-2524.

[9] A. Tang, J. W. Lee, J. Huang, M. Chiang, and A. R. Calderbank, "Reverse engineer MAC," in Proc. WiOpt, 2006.

[10] H. Inaltekin and S. B. Wicker, "The analysis of Nash equilibria of the one-shot random-access game for wireless networks and the behavior of selfish nodes," IEEE/ACM Trans. Netw., vol. 16, no. 5, pp. 1094-1107, Oct. 2008.

[11] I. Aad, Q. Ni, C. Barakat, and T. Turletti, "Enhancing IEEE 802.11 performance with slow CW decrease," in Proc. IEEE INFOCOM, 2001.

[12] Y. Kwon, Y. Fang, and H. Latchman, "A novel MAC protocol with fast collision resolution for wireless LANs," in Proc. IEEE INFOCOM, 2003, vol. 2, pp. 853-862.

[13] G. Bianchi, L. Fratta, and M. Oliveri, "Performance evaluation and enhancement of the CSMA/CA MAC protocol for 802.11 wireless LANs," in Proc. IEEE PIMRC, 1996, vol. 2, pp. 392-396.

[14] F. Cali, M. Conti, and E. Gregori, "Dynamic tuning of the IEEE 802.11 protocol to achieve a theoretical throughtput limit," IEEE/ACM Trans. Netw., vol. 8, no. 6, pp. 785-799, Dec. 2000.

[15] IEEE 802.11 Task Group E [Online]. Available: http://grouper.ieee. org/groups/802/11/Reports/tge_update.htm

[16] M. Heusse, F. Rousseau, R. Guillier, and A. Dula, "Idle sense: An optimal access method for high throughput and fairness in rate diverse wireless LANS," in Proc. ACM SIGCOMM, 2005, pp. 121-132.

[17] C. Hu and J. C. Hou, "A novel approach to contention control in IEEE 802.11e-opereated WLANS," in Proc. IEEE INFOCOM, 2007, pp. $1190-1198$.

[18] R. Abraham, J. E. Marsden, and T. Ratiu, Manifolds, Tensors, Analysis, and Applications, 2nd ed. New York: Springer-Verlag, 1988.

[19] D. Fudenburg and J. Tirole, Game Theory. Cambridge, MA: MIT Press, 1991.

[20] D. P. Bertsekas and J. N. Tsitsiklis, Parallel and Distributed Computation. Englewood Cliffs, NJ: Prentice-Hall, 1989.

[21] L. Chen, S. Low, and J. Doyle, "Contention control: A game-theoretic approach," in Proc. IEEE CDC, 2007, pp. 3428-3434.

[22] S. D. Flam, "Equilibrium, evolutionary stability and gradient dynamics," Int. Game Theory Rev., vol. 4, no. 4, pp. 357-370, Dec. 2002.
[23] G. Bianchi, "Performance analysis of the IEEE 802.11 distributed coordination function," IEEE J. Sel. Areas Commun., vol. 18, no. 3, pp. 535-547, Mar. 2000.

[24] A. Kumar, E. Altman, D. Miorandi, and M. Goyal, "New insights from a fixed point analysis of single cell IEEE 802.11 WLANS," in Proc. IEEE INFOCOM, 2005, vol. 3, pp. 1550-1561.

[25] D. Bertsekas and R. Gallager, Data Networks, 2nd ed. Englewood Cliffs, NJ: Prentice-Hall, 1992.

[26] R. Jain, D. Chiu, and W. Hawe, "A quantitative measure of fairness and discrimination for resource allocation in shared computer systems," DEC, Res. Rep. TR-301, 1984.

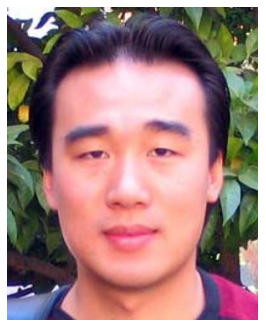

Lijun Chen (M'05) received the B.S. degree in physics from the University of Science and Technology of China, Hefei, China; the M.S. degree in theoretical physics from the Institute of Theoretical Physics, Chinese Academy of Sciences, Beijing, China, and from the University of Maryland at College Park; and the Ph.D. degree in control and dynamical systems from California Institute of Technology (Caltech), Pasadena.

$\mathrm{He}$ is a Research Scientist with the Control and Dynamical Systems Department, Caltech. His current research interests are in communication networks, optimization, game theory, and their engineering application.

Dr. Chen was a co-recipient of the Best Paper Award at the IEEE International Conference on Mobile Ad-hoc and Sensor Systems (MASS) in 2007.

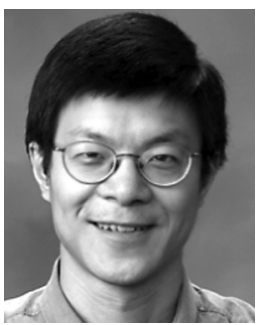

Steven H. Low (F'08) received the B.S. degree from Cornell University, Ithaca, NY, and the Ph.D. degree from the University of California, Berkeley, both in electrical engineering.

$\mathrm{He}$ is a Professor with the Computer Science and Electrical Engineering departments at California Institute of Technology, Pasadena, and an Adjunct Professor with Swinbourne University, Melbourne, Australia. Prior to that, he was with AT\&T Bell Laboratories, Murray Hill, NJ, and the University of Melbourne, Melbourne, Australia.

Prof. Low was a co-recipient of the IEEE Bennett Prize Paper Award in 1997 and the 1996 R\&D 100 Award. He was a member of the Networking and Information Technology Technical Advisory Group for the US President's Council of Advisors on Science and Technology (PCAST) from 2006 to 2007. He was on the Editorial Board of the IEEE/ACM TRANSACTIONS ON NETWORKING from 1997 to 2006 and the Computer Networks Journal from 2003 to 2005. He is currently on the editorial boards of Computing Surveys and Foundations and Trends in Networking and is a Senior Editor of the IEEE JOURNAL ON SELECTED AREAS IN COMMUNICATION.

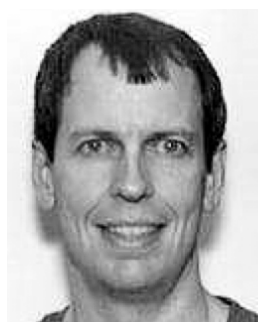

John C. Doyle received the B.S. and M.S. degrees in electrical engineering from Massachusetts Institute of Technology, Cambridge in 1977, and the Ph.D. degree in math from the University of California, Berkeley, in 1984.

He is John G. Braun Professor of control and dynamical systems, electrical engineering, and bioengineering at California Institute of Technology, Pasadena. His group has contributed to many software projects, including the Robust Control Toolbox (muTools), SOSTOOLS, Systems Biology Markup Language (SBML), and Fast AQM, Scalable TCP (FAST). His current research is in theoretical foundations for complex networks in engineering and biology, focusing on architecture, and for multiscale physics. His early work was in the mathematics of robust control, including recent extensions to nonlinear and hybrid systems.

Prof. Doyle has received the IEEE W. R. G. Baker Award, the IEEE Transactions on Automatic ConTrol George S. Axelby Award (twice), and Best Paper awards at the ACM SIGCOMM and AACC American Control conferences. His individual awards include the AACC Eckman Award, and the IEEE Control Systems Field Award, and the IEEE Centennial Outstanding Young Engineer Award. 